\title{
Atomic Force Microscopy on Cross Sections of Fuel Cell Membranes, Electrodes, and Membrane Electrode Assemblies
}

Renate Hiesgen $^{\mathrm{a}^{*}, 1}$, Tobias Morawietz $^{\mathrm{a}}$, Michael Handl ${ }^{\mathrm{a}}$, Martina Corasaniti ${ }^{\mathrm{b}}, \mathrm{K}$. Andreas Friedrich ${ }^{\mathrm{c}, 1}$

a University of Applied Sciences Esslingen, Kanalstrasse 33, Esslingen 73728, Germany

b Solvay Specialty Polymers Italy S.p.A., Viale Lombardia, 20, 20021 Bollate (Mi), Italy

c German Aerospace Center, Institute of Technical Thermodynamics, Pfaffenwaldring 38-40, Stuttgart 70569, Germany

1 ISE member

* $\quad$ Author to whom correspondence should be addressed:

E-Mail: renate.hiesgen@hs-esslingen.de

Dedicated to Prof. Jacek Lipkowski, in recognition of his achievements and on the occasion of his 70th birthday.

Keywords: AFM; PFSA; conductive ionic network; fuel cell catalytic layer; ionomer content

\section{Abstract}

Using material-sensitive and conductive atomic force microscopy (AFM) on cross sections of perfluorinated and sulfonated membranes at low humidity, crystalline polymer lamellae were imaged and their thickness determined to approximately 6 $\mathrm{nm}$. In the capacitive current, water-rich and water-poor areas with different phase structures were investigated. The formation of a local electrochemical double layer within the water-rich ionically conductive areas at the contact of the AFM tip with the electrolyte enabled their visibility in adhesion force images. The large water-filled ionically conductive areas include numerous ionic domains. Under equilibrium conditions, these areas are spherical (appearing circular in the images) and with distinct size distribution. Forcing a current through the membranes (current-induced 
activation) led to merging of the water-filled ionically conductive areas in the voltage direction and resulted in an anisotropic ionically conducting network with flat channels. The distribution of the current in the membrane and catalytic layers of a pristine membrane electrode assembly (MEA) was analyzed. From the adhesion force mappings, an inhomogeneous distribution of ionomer in the catalytic layer was detected. Cross currents between Pt/C particles through large ionomer particles within the catalytic layer were detected and the ionomer content across an electrode was evaluated.

\section{Introduction}

For polymer membrane fuel cell application mostly Nafion ${ }^{\circledR}$ from DuPont is used as electrolyte, but recently the importance of related materials such as AQUIVION ${ }^{\circledR}$ is increasing. The two ionomer materials were used as membranes and as ionically conductive components within the electrode. Nafion $^{\circledR}$ and AQUIVION ${ }^{\circledR}$ are both perfluorinated polymers with sulfonated end groups at their side chains (PFSA). These materials differ in the length of their side chains, which leads to somewhat different properties based on the different nanostructures [1],[2]. Together with water, these molecules undergo a phase separation upon solidification; the sulfonated end groups cluster together to form a hydrophilic ionic phase, which is the basis of a continuous ionically conductive network. The polymer main chains also cluster together to form bundles with a size of a few nanometers [3] and provide mechanical strength. Approximately $20 \%$ of the fluorocarbon chains are crystalline [4], thus contributing to the mechanical strength. The size of ionic phase structures has been intensively studied using scattering techniques such as small angle X-ray scattering (SAXS). A strong effect on the water content and pretreatment is generally observed, monitored by the shift of the generally broad peaks, mainly the so-called ionic peak. 
SAXS results indicate a value of 3 to $7 \mathrm{~nm}$, which is attributed to the distance of the ionic areas [5]. Nanostructure models that fit the measured spectra for a wide range of humidity and temperature values exist; however, no consensus has been reached on the precise nanostructure.

Due to the high sensitivity of PFSA toward humidity changes, the structure determination is highly complex and controversial results have been published. It has to be noted that changes occur with time constants that comprise several orders of magnitude [6]. In addition, the structure under equilibrium and the structure under non-equilibrium conditions, such as the current flow, may differ greatly. The protons that carry the ionic current are solvated, and depending on the conditions, one proton drags an average of 1-3 water molecules during fuel cell operation (electro-osmotic drag and permeation, see [7] and values therein). In addition, the current water exerts a mechanical pressure on the polymer.

Despite the self-assembly of the two different phases, the hydrophilic and the hydrophobic phase are a prerequisite for the formation of a continuous ionically conducting network; it may not exist in pristine PFSA, at least at lower humidity. In a previous study it was demonstrated that for the induction of a faradaic current a forced current flow was necessary, called the current-induced activation of the membrane [8]. Investigation of the PFSA structure is therefore assumed to yield different resulting nanostructures depending whether equilibrated samples or samples under or after current flow, as necessary for fuel cell operation, are analyzed.

In previous studies, mostly samples at different equilibrium states have been studied and compared to various nanostructure models. A detailed discussion of existing models can be found in [1]. Nafion ${ }^{\circledR}$ was first described by Gierke et al. [9], with a model of ionic clusters dispersed in a hydrophobic polymeric matrix. This model was 
based on the reduction of the total free energy of the polymer system, described by Eisenberg [10]. Based on the experimentally observed linear volume change with humidity, a lamellar stacking of fluorocarbon chains with ionic side groups that sandwich water layers with various thicknesses was proposed by Falk et al. [11] and Litt [12], and was further refined by Haubold [13]. Recently, Kreuer et al. predicted a lamellar structure of perfluorinated membranes, based on the experimental findings in combination with electrostatic considerations [5]. In our previous study at low humidity, we imaged for the first time a lamellar stacking of Nafion ${ }^{\circledR}$ and AQUIVION ${ }^{\circledR}$ PFSA in AFM experiments [14].

An alternative model in the literature consists of flat polymeric fibrils and bundles of aggregated polymer backbones with a bundle size of approximately 3-4 nm, aggregated to aligned crystalline structures of approximately $50 \mathrm{~nm}$ based on the scattering data proposed by Gebel et al. [15],[16],[17]. In this model, water surrounds the fibrillar objects and is not confined to pores. Predictions from modeling also provide input on the discussion, and recent models combine aspects from various existing models to suggest a more complicated structure, including independent bicontinuous networks of ionic clusters and fluorocarbon chains [18]. For the application of PFSA membranes in fuel cells, the ionomer/electrode interfaces play an important role because this is the location of the electrochemical reaction, and it may limit the performance of the cell. At the surface of PFSA membranes in air, the existence of a surface skin layer has been proposed by several authors and is currently accepted [19],[20]. At equilibrium conditions, this layer forms due to the different surface energies of the membrane and environment. At the membrane/air interface, a hydrophobic surface is formed. From GISAX measurements, a high crystallinity of a layer of an approximately 5-nm layer with an orientation parallel to the surface was observed [21]. From other scattering 
experiments, including synchrotron grazing incidence X-ray diffraction (GIXRD) and small angle X-ray scattering (GISAXS) analyses, a similar value was obtained [21],[22]. Recently, AFM measurements confirmed this thickness for the skin layer for membranes in air [23]. This highly adhesive surface phase is fluorocarbon-rich with the ionic side groups folded inwards [24]. Consequently, the surface properties of the outer surface and cross section are expected to differ significantly.

For the investigation of the PFSA nanostructure, scanning probe techniques are well suited [25],[26],[27],[28]. With advanced tapping mode techniques, the differentiation between different materials, including different phases, allows a direct imaging of nanostructure under various temperature and humidity conditions. Using conductive AFM, analysis of the structures under non-equilibrium conditions as current flow has been conducted [8],[23],[29],[30],[31],[32]. The disadvantage of this approach is the restriction of the measurement to surface or subsurface properties and the dependence of the obtained resolution on the tip size and shape.

The electrodes of a fuel cell fulfill several functions, including high catalytic activity but also the provision of electronic and ionic conductivity and the appropriate transport of water and reactant gases. This complex functionality is normally achieved by mixing ionomer with the catalytically active nanometer platinum particles supported on carbon. The size and distribution of the components, especially of the ionomer in the catalyst layer, determines the performance and stability of the electrodes, i.e., based on the volume of the electrochemically active catalyst area, which affects the proton conductivity, mass transport, electronic conductivity, and porosity [33].

For a quantitative analysis of the electrode components, a method with a high lateral resolution is needed to image the nanosized particles and obtain a quantitative result of the composition. In the past, the ionomer content within the electrodes was mostly 
analyzed using transmission electron microscopy (TEM)[34][35] [36] in combination with energy dispersive elementary analysis (EDX) or energy filtering to obtain a mapping of the fluorine content. TEM-based techniques require very thin slicing and staining of the sample below $100 \mathrm{~nm}$. For these techniques, staining with heavy metal atoms is often applied to enhance the scattering contrast. Artefacts such as swelling, or pore penetration with epoxy fillers may occur. Ionomer damage may occur due to electron beam radiation. The ionomer volume may shrink and change its structure due to water loss in the vacuum, a problem only avoided using cryo-TEM [37]. Modern scanning electron microscopy with increasing resolution is also able to image electrodes, including the ionomer; however, the issues of polymer radiation damage and water loss are similar to those encountered for TEM analysis, and quantitative analysis may be difficult to circumvent.

Recently, the analysis of components in fuel cell has been performed using synchrotron based soft X-ray scanning transmission microscopy (STXM) [33], [38],[39]. The chemical contrast between the elements is facilitated using the near edge X-ray absorption with a spatial resolution of $30 \mathrm{~nm}$. For the samples, 100-300$\mathrm{nm}$ thick ultra-microtome cut slices of membrane electrode assembly (MEA) samples were used. STXM has been used for characterizing ionomer distributions in differently applied catalyst layer structures, yielding quantitative maps of the ionomer and carbon support components [40]. Although some radiation damage is still detectable for radiation sensitive polymers such as PTFE, the advantage over energy-filtered TEM is significant using the same radiation dose. In this study, we have two different sections. First, we employ material-sensitive and conducting AFM to analyze the structure, phase separation, and development of the continuously connected ionically conducting phase in Nafion ${ }^{\circledR}$ and AQUIVION ${ }^{\circledR}$ PFSA membranes. Here, we use AFM for detection of properties that influence the function 
of a fuel cell: the conductive structure of the membrane with the detection of waterrich phase using membrane cross sections and the formation of the ionically conducting network under forced current flow; the interface of the membrane which impacts the interface to the electrode and inside the electrode to the $\mathrm{Pt} / \mathrm{C}$ particles with an investigation of the ionomer/ionomer interface formation and its interface properties. In the second part we report on the application of AFM for MEAs and we can clearly distinguish ionomer phase in electrodes for the first time with this method with a resolution in the nanometer range. The structure and conductivity of pristine MEA cross-sections and catalyst layers were investigated. Material-sensitive AFM was used to statistically evaluate the ionomer content in the electrodes and to obtain insight into the conductivity of the embedded ionomer particles.

\section{Experimental}

\subsection{Atomic force microscopy}

For the atomic force microscopy (AFM) investigation, a Bruker Multimode 8 AFM (Karlsruhe, Germany) equipped with a Nanoscope $\vee$ controller, a closed loop scanner with open loop Z-axis (nPoint, USA), quantitative nano-mechanical tapping mode (QNM ${ }^{\mathrm{TM}}$, Bruker Corp.), current detection (PeakForce-TUNA ${ }^{\mathrm{TM}}$, Bruker Corp.), and a gas tight chamber was used. The current was measured in Peakforce TUNA tapping mode and averaged using a lock-in amplifier (PF-TUNA Module, Bruker). The measurement of polymer components was performed with PtIr-coated tips (Olympus AC240TM, 2 N/m; Olympus; PPP-NCHPt, 42 N/m; Nanosensors). Low spring constant probes were used for higher resolution, and high spring constant probes were used to image the stiffer parts of the electrode that contain carbon and platinum. 
The AFM measurements were performed in the quantitative nano-mechanical mode $\left(\mathrm{QNM}^{\mathrm{TM}}\right.$, Bruker Corp.). During scanning, the tip follows a sinusoidal path along the sample and touches the surface once every cycle to record an image point. During forward scanning and the retrace to the surface, mechanical properties are retrieved from the force-separation behavior simultaneously with the height information. As a consequence, the adhesion force, stiffness (Derjaguin, Müller, and Toporov (DMT) modulus [41]), deformation, and energy dissipation mappings are recorded. Quantitative measurement is possible using a calibration procedure with a suitable reference sample. For the ionomer samples, a phase-separated mixture of polyethylene and polystyrene was used.

The continuous ionic current flow detected in a humid environment at the ionomer membrane is based on two electrochemical reactions, essentially oxygen reduction and oxygen evolution at the extended porous Pt-containing back electrode and at the platinum-coated AFM tip. The latter works as cathode for the back reaction [29]. For ionic current flow the bias voltage must exceed 1.4 V. The tapping mode current measurements were performed in PeakForce-TUNA ${ }^{\mathrm{TM}}$ mode. This mode enables simultaneous retrieval of nano-mechanical information and the current. The current signal was averaged using a lock-in amplifier. If not otherwise stated, the samples for current measurements were activated in an electrolysis setup at $2.8 \mathrm{~V}$. Details are given in [23]. After activation the samples were stored in ultra-pure water (Millipore, $18 \mathrm{M} \Omega$ ). As a water reservoir for the current measurements, a droplet of ultra-pure water was placed in front of the sample to guarantee a sufficiently high humidity of the membrane and electrodes for ionic current flow. For recording the capacitive current, a bias voltage of approximately $100 \mathrm{mV}$ to $2 \mathrm{~V}$ was applied. The electronic current flow at an electrode was measured at a bias voltage of $\mathrm{U} \sim 20-100 \mathrm{mV}$. 


\subsection{Sample preparation}

For investigation of the interfaces, ionomer samples cast from aqueous AQUIVION ${ }^{\circledR}$ PFSA D83-06A dispersion were prepared as model layers. The layers were applied by doctor-blade coating and were dried at ambient humidity at room temperature for one day. After drying, the sample was embedded between two polystyrene sheets. Cross sections of the samples were prepared by a microtome (Leitz microtome type 1310, Germany) and fixed to a steel sample holder with adhesive tape without touching the surface. If needed, silver containing glue was used to enhance the electrical contact. The membrane samples were pieces cut from commercial membranes of Nafion ${ }^{\circledR} \mathrm{NR} 212$ (equivalent weight of $1100 \mathrm{~g} \cdot \mathrm{eq}^{-1}$ ) with a membrane thickness of $50 \mu \mathrm{m}$, an electrode thickness of $10 \mu \mathrm{m}$, and a gas diffusion layer (GDL) SGL 25BA with $190 \mu \mathrm{m}$ (Figure 7). If not otherwise stated, the samples were equilibrated in air with approximately $30-40 \%$ relative humidity (rh). A few measurements were performed at higher humidity in a gas-tight chamber using a humidifier. A relative humidity less than $30-40 \%$ was achieved by purging the chamber with dry Argon. Specified values for the equilibration conditions and the relative humidity during the measurement are given for each figure.

In addition to the membranes, membrane electrode assemblies (MEA) were investigated. The enclosed membrane consisted either of reinforced AQUIVION ${ }^{\circledR}$ PFSA (Figure 9) or a reinforced Nafion ${ }^{\circledR} \mathrm{XL}$ membrane with a thickness of $24.5 \mu \mathrm{m}$ and 7- $\mu \mathrm{m}$-thick electrodes without GDL. The electrodes had a platinum loading of 0.4 $\mathrm{mg} / \mathrm{cm}^{2}$ on the cathode and $0.2 \mathrm{mg} / \mathrm{cm}^{2}$ on the anode (Figures 10-15). No additional pretreatment was applied. The MEA samples were cut as delivered by the supplier. For AFM measurements of the cross sections the samples were cut using a microtome and embedded between two plates of polystyrene. Before being cut, double-sided conductive tape was placed on top of the sample sandwich to stabilize 
the cross section after cutting. The slices were then fixed to a magnetic steel disc AFM sample holder without touching the surface (Figure 1).

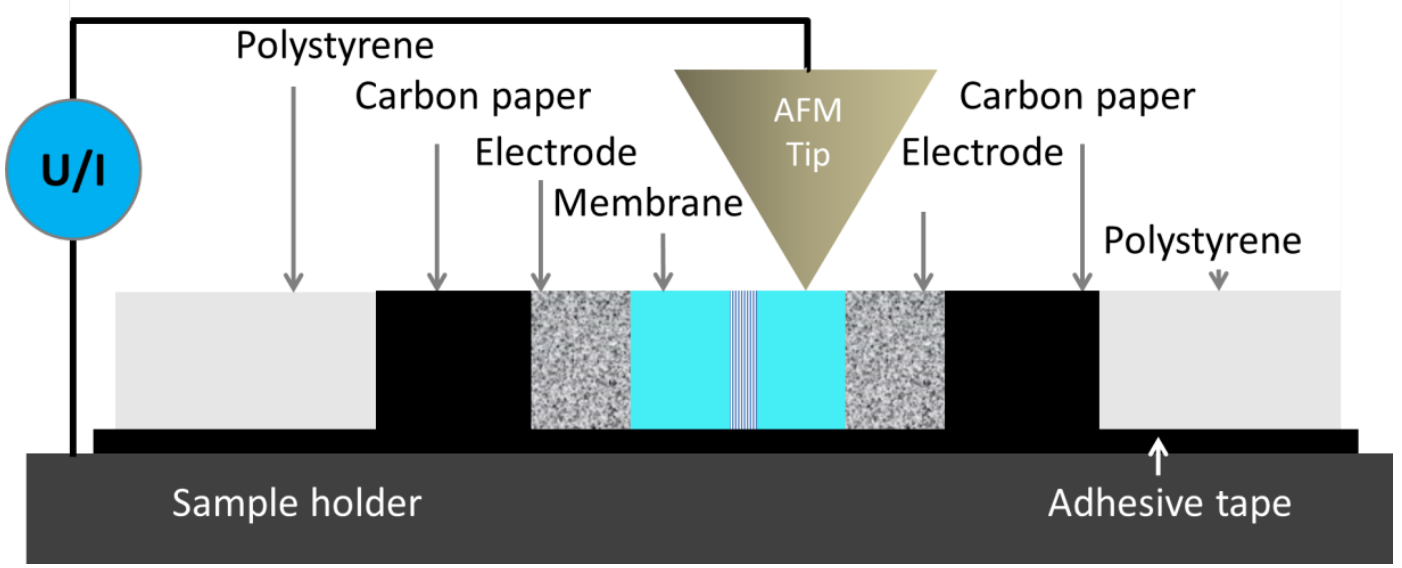

Figure 1: Scheme of cross section of MEA for current measurement.

\section{Results and Discussion}

\subsection{Material identification by material-sensitive and conductive AFM}

\subsubsection{Structure of Nafion ${ }^{\circledR}$ membrane surface and cross section}

Because of the existence of a surface skin layer [8] [21], freshly cut cross sections of Nafion $^{\circledR}$ and AQUIVION ${ }^{\circledR}$ PFSA were investigated by AFM in order to obtain information on the bulk structure.

\subsubsection{Structure of Nafion ${ }^{\circledR}$ membrane surface and cross section}

The surface of the freshly cut cross section, equilibrated and measured at $30-40 \% \mathrm{rh}$ without further pretreatment, contained sizeable ordered regions as observed in Figure 2. The 3D topography of the 570-nm-wide image (Fig. 2a) exposes a terraced surface composed of layers or lamellae structures [14]. By overlaying the peak force control signal onto the topography in Figure $2 \mathrm{~b}$, the lamellar edges are enhanced for better visibility. The simultaneously recorded mapping of the adhesion force overlaid 
on topography in Figure $2 \mathrm{c}$ reveals two distinct phases with significantly different adhesion. After one hour of scanning, the existence of at least a thin hydrophobic skin layer must be assumed (compare chapter 3.1 .3 ) but no obvious change was observed in the large low-adhesive areas.
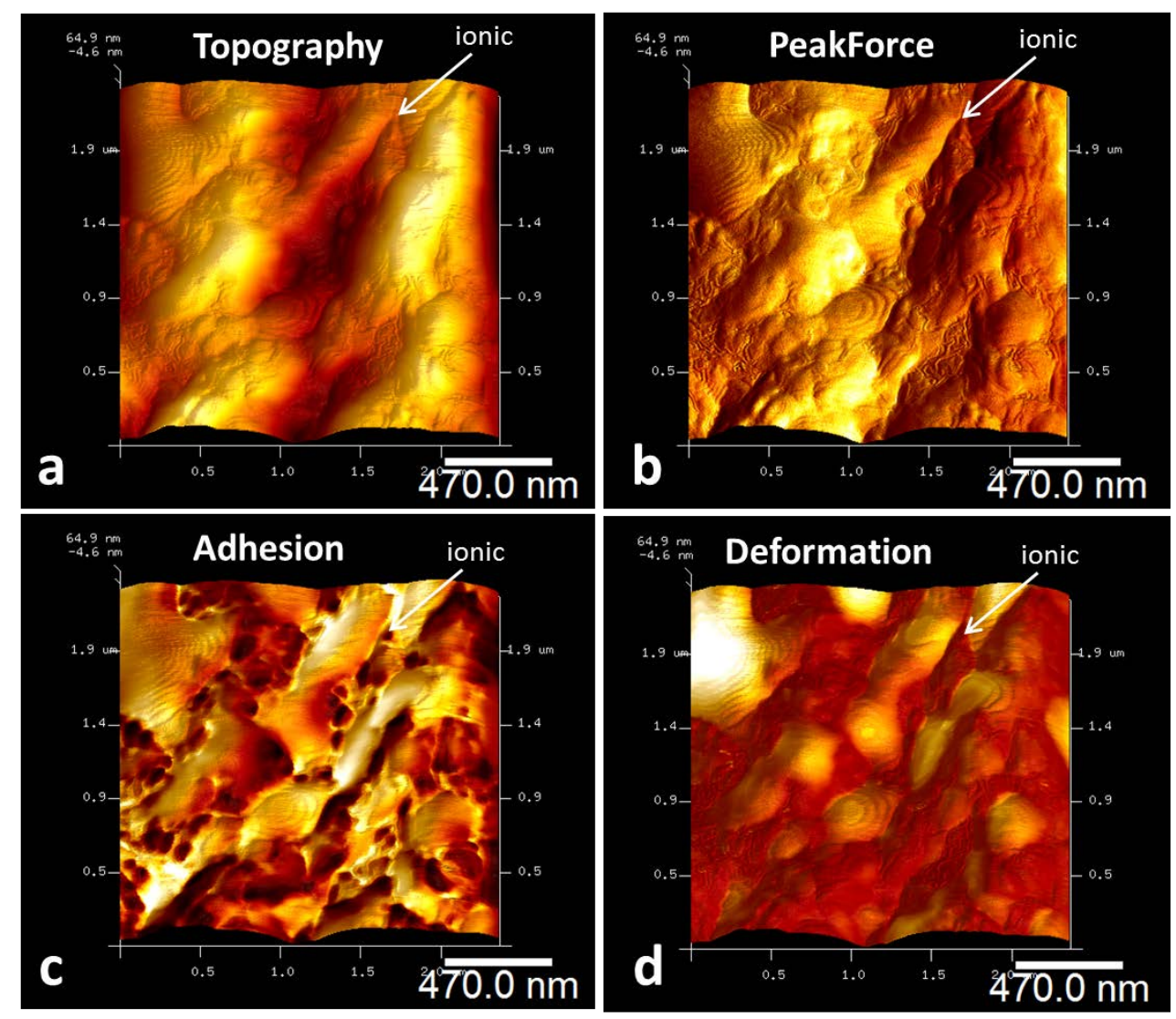

Figure 2: Cross section of non-activated Nafion ${ }^{\circledR} 212$, equilibrated and measured at 30-40 \% rh: (a) 3D topography, (b) peak force control signal overlaid on topography, (c) adhesion force mapping overlaid on topography, and (d) deformation mapping overlaid on topography.

From previous experiments, the terraced, bright, high-adhesive phase can be attributed to higher fluorocarbon chain (PTFE-like) content, and the embedded dark, circular, low-adhesive phase can be attributed to a region of water-filled interconnected ionically conductive phase [8]. Additional ionic areas with low water content that are not connected but must be present all over the sample, do not lead to a visible change in the adhesion contrast. The size of these circular water-filled 
structures is typically $30-50 \mathrm{~nm}$ in diameter, with most of the structures being similar in size. The lower deformation (Figure 2d) and higher stiffness (not shown here) at these positions, which were also observed in previous studies, were attributed to a high internal pressure in the ionic network resulting from the water pressure [8].

The terraced back-bone-rich phase reveals a high degree of ordering. As demonstrated in a previous study [14], this phase consists of lamellar stacking of backbone sheets and ionic side groups. A comparison of the lamellar heights of Nafion ${ }^{\circledR}$ and AQUIVION $^{\circledR}$ PFSA, a shorter side chain molecule, revealed in both cases a layer thickness comparable to that of a molecular bilayer, estimated with half-stretched side chains to $1.7 \mathrm{~nm}$ and $1.5 \mathrm{~nm}$, respectively [14]. In the other lateral direction a layer thickness of roughly $6 \mathrm{~nm}$ has been determined. In a study of the crystallinity in cast Nafion ${ }^{\circledR}$, Ludvigsson et al. determined a spherulitic morphology for Nafion ${ }^{\circledR}[4]$. They emphasized that the spherulites grown from the bulk consist of sheets build of zig-zag-folded polymer chains, which grow perpendicular to the film surface, a fact that prevented their determination of the lamellar thickness in this study. The growth of such crystalline lamellae has been studied for different polymers and the formation of crystalline sheets has been described in a general law by Strobel [42]. For polyethylene and Nafion ${ }^{\circledR}$, Ludvigsson et al. reported a similar structure based on $x$-ray investigation. The varying thickness of such sheets measured by AFM was in the order of $10 \mathrm{~nm}$ in PE and $20 \mathrm{~nm}$ in s-PP-20 nm [43]. Following these studies, we assign the observed lamellar structures also to crystalline sheets. In the plane of folded zig-zag polymer strains, the distance of backbone layers is determined by the two side chains as was assumed in our earlier work [14]. The larger steps with roughly $6 \mathrm{~nm}$ thickness are associated to the polymer crystalline lamellae thickness. These layers may include different amounts of water bound to the side chain sulfonic acid groups and the water amount depends strongly 
on humidity. The resulting varying layer distance may limit the visibility of this phase in scattering analysis.

\subsubsection{Formation of a new skin layer at cross section}

The freshly cut surface of cross section exposed to air is not in equilibrium and dependent on relative humidity immediately starts to form a new surface skin layer. It is known from the literature that the sulfonic acid groups at the side chains quickly, probably within seconds, turn to the interior and the surface properties change from hydrophilic to hydrophobic [24]. Due to the transfer of the cut sample to the AFM we can follow these changes only after the first 2-10 minutes depending on handling time. The change of adhesion and stiffness values was measured using a suitable tip for adhesion and stiffness detection. The images were continuously scanned with a high speed. As a measure for the stiffness, the peak value of the distribution was taken; for the adhesion the percentage of the area above a fixed adhesion threshold was evaluated. A typical time dependence of area with high adhesion and stiffness values is given in Figure 3a. Both samples were equilibrated at $40 \%$ rh. At a relative humidity of $40 \%$ during measurement the high-adhesion area decreased during the first 20 min and then started to rise again. The stiffness values measured at $50 \%$ rh first rose for a few minutes before they decreased after about 20 min. From the literature it is known that there is a high internal pressure inside the ionomer membrane [19]. We interpret the rise of stiffness by an increased surface pressure, caused by the transport of water to the open interface after cutting. The subsequent decrease of stiffness is interpreted by a decrease of hydrostatic pressure with time, due to water evaporation. The decrease of area with high adhesion after cutting is caused by the loss of humidity at the interface that reduces the area with high adhesion. The subsequent increase of high-adhesive area is explained by the 
formation of PTFE-rich surface areas that have a higher adhesion to the tip [31] than the dry ionic surface. In Figure $3 \mathrm{~b}$ measurements of the time-dependent stiffness values for cross section of a sample at $70 \%$ rh are shown for an extended time period. Again the above described dependence is seen and after one hour of scanning, the stiffness values of the sample measured under these conditions slowly increased again. This can be explained by the increased loss of water from the surface that led to a formation of a hydrophobic skin layer. The water-poor layer inhibits the further evaporation of water and again leads to an increased hydrostatic pressure. However, some details of the time dependence which are reproducible cannot be explained and need further investigation.

As a consequence of these investigations, always a fresh cut cross section was used except otherwise stated, and it was taken care that during the measurement the surface properties did not change significantly.

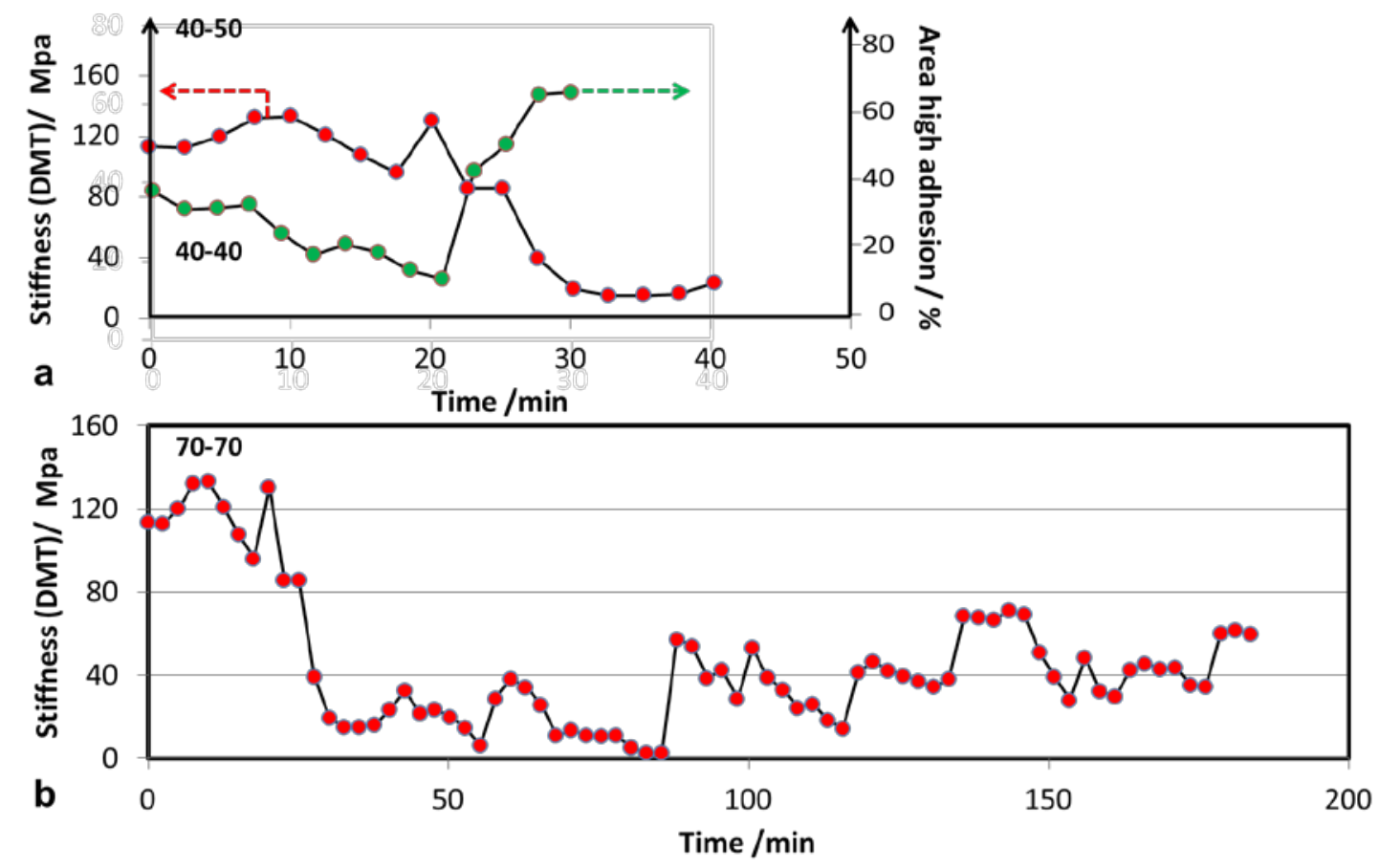

Figure 3: Time dependence of high-adhesive area and stiffness values at a cross section of Nafion $212^{\circledR}$ after cutting: (a) stiffness values (red circles) and adhesive area (green circles) for samples equilibrated at $40 \%$ rh and measured at $50 \%$ and 
$40 \%$, respectively; and (b) time dependence of stiffness values of two different samples measured at different days, equilibrated at $70 \%$ rh and measured at $25 \%$ rh (blue circles) and $70 \%$ rh (red circles).

\subsubsection{Conductivity}

Using conductive AFM, two different current contributions at PFSA cross sections were detected. A capacitive current is present in most cases, whereas a steady-state faradaic current is observed mainly at activated membranes and requires two electrochemical reactions at the two electrodes in the set-up. When the AFM tip approaches the proton-conducting electrolyte surface, an electrochemical doublelayer is formed. Therefore, both scanning the tip across the membrane and tapping toward the surface, will induce a capacitive current based on the differential capacitance and the scan rate [14] even without the presence of catalytic back electrodes. The change of the capacitive current contribution of $i(V)$ curves at different scan rates with the same tip area is shown in Figure 4. These experiments were performed without platinum in the back electrode. The current recorded with the same tip is proportional to the current density. The current difference $\Delta i$ varies linearly with the scan rate, as observed in Figure $4 \mathrm{~b} . \Delta \mathrm{i}$ was measured between the negative and positive scan direction of the $\mathrm{i}(\mathrm{V})$ curve at the same voltage (marked in Figure 4a). The sample voltage scan deviates from the potential scale indicating an additional set-up induced resistance that decreases at higher humidity. The tip is biased through the sample and at negative voltages hydrogen evolution at the tip is visible whereas at positive voltages platinum oxidation can be seen in the forward and the reduction in the backward scan direction. An example of a current measurement where only a capacitive current contribution was recorded is given in Figure 4c. In the histogram, the percentage of current values of the total number of 
pixels in the current image is plotted versus current. The capacitive current contribution has values of only a few $\mathrm{pA}$.

A faradaic current contribution can only be observed if the tip is in contact with a continuous ionically conducting network under humid conditions. At suitable potentials, the electrochemical reactions at the two electrodes drive a current through the sample. The resulting faradaic current contribution is shown in the current histogram in Figure $4 \mathrm{~d}$. A scheme that illustrates the isolated water-filled ionic regions with only capacitive current is given in Figure $4 \mathrm{e}$, and a scheme that illustrates the continuous ionic connection between electrodes for faradaic current flow is given in Figure $4 f$.
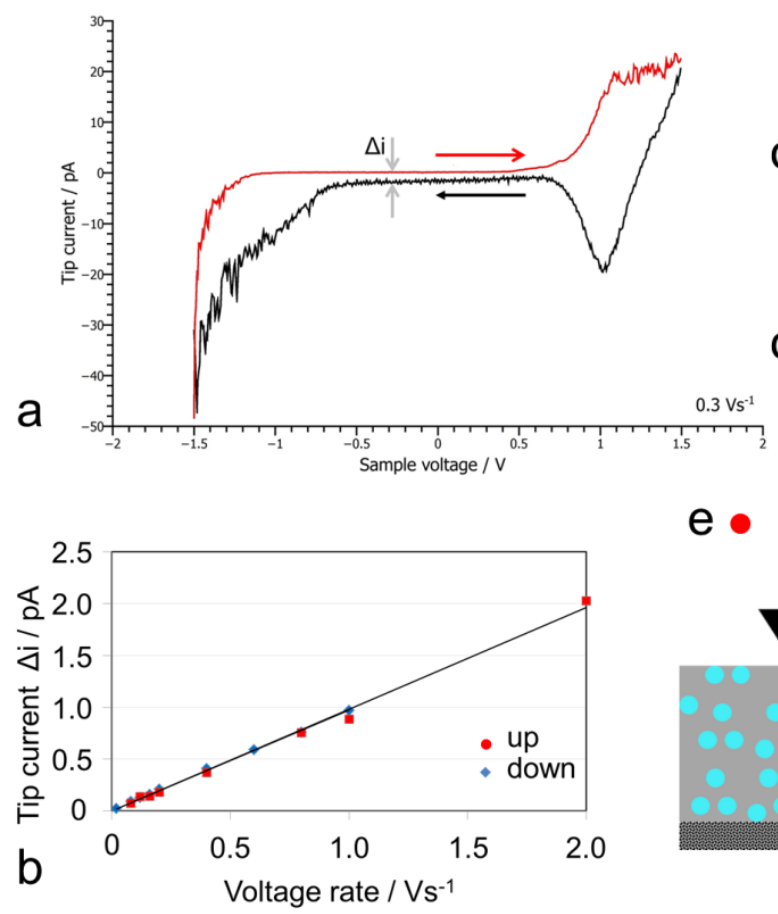
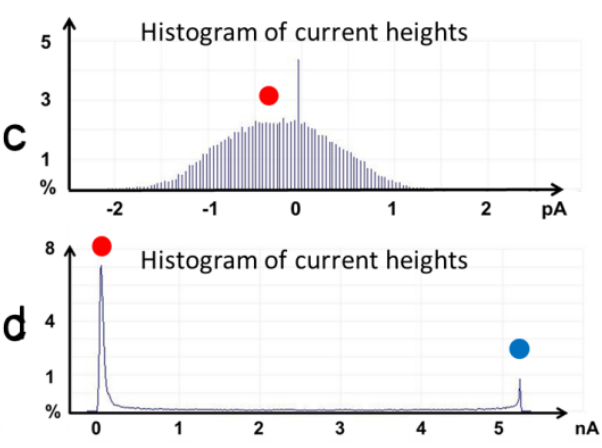

e

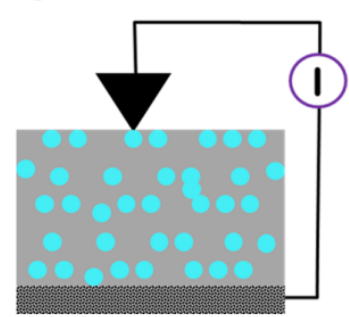

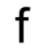

$f$

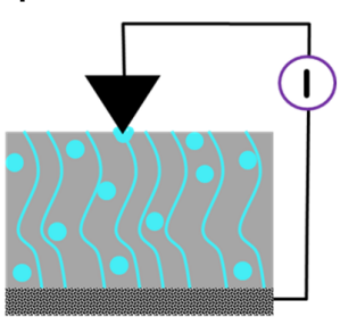

Figure 4: (a) I (v) curve measured at a scan rate of $0.3 \mathrm{~V} \cdot \mathrm{s}^{-1}$ for wet activated Nafion ${ }^{\circledR} 212$ in air without catalytic back layer and with constant tip area, (b) dependence of current hysteresis $\Delta i$ as a measure of the capacitive current with varying scan rate, (c) histogram of capacitive current, (d) histogram of capacitive and faradaic current after activation, (e) scheme that shows isolated water-filled ionic regions with only capacitive current, and (f) scheme that illustrates the continuous ionic connection between electrodes for faradaic current flow. 
In addition to a few defects that allow water exchange, the hydrophobic surface skin layer inhibits the direct connection of the ionic conducting water-rich phase inside the membrane with the environment [19]. The formation of different surface structures under dry and wet conditions has been investigated with two subsequently applied ionomer layers and is described in chapter 3.2. For pristine membrane samples, no continuous current flow can be detected by AFM [23]. Even in case a continuous ionically conducting network to the back electrode exists, the hydrophobic skin layer inhibits a direct conductive connection [19]. By scanning the tip across the surface, only a small capacitive current on the order of a few $\mathrm{pA}$ is typically present. A conductivity increase of several orders of magnitude is observed only after current flow is forced through the sample, called current-induced "activation" [31], [8].

\subsubsection{Equilibrium structure of ionomer}

In pristine ionomer membranes before any current flow, the water-filled interconnected ionic areas, identified by their low adhesion, were isolated as visible in Figure 2. Their distribution was inhomogeneous, and they typically had a circular shape. These low-adhesive regions have a typical size at the surface of a few tens of nanometers, often between 30 to $50 \mathrm{~nm}$, as observed in Figures 2 and 5. The lower adhesion cannot be explained by the adhesion of the topmost surface. One must assume that at least a thin hydrophobic surface layer is present after one or two hours of scanning as discussed above. A decreased adhesion measurement can be explained by an additional repulsive force acting on the tip, either a long-range force that is not much influenced by a thin surface layer or a repulsive mechanical contact force, i. e., imposed by a high surface stiffness. The stiffness response depends on humidity and measurement conditions, for example with water transport under current flow, the low-adhesive areas can have a high stiffness, but at humidity 
equilibration a low stiffness is observed in those areas. Therefore the low adhesion of the water-filled ionic areas cannot be explained by mechanical interactions alone but an influence of the electrochemical double layer formation at the tip-sample contact, which leads to a repulsive (coulomb-) force toward the tip is deduced. In the forceseparation curve, this repulsive force appears as reduced adhesion. In the topography image (Figure 2a), $1-\mu m^{2}$ area of a freshly cut cross section of Nafion ${ }^{\circledR}$ 212 , equilibrated and measured at $30-40 \%$ rh is shown. The corresponding adhesion image (Figure $2 b$ ) reveals numerous statistically distributed circular low-adhesive ionic areas. It is assumed that water uptake does not lead to an even distribution of water in the membrane. The water is expected to cluster forming droplets due to an ionomer matrix which has on average a much lower surface energy. The size of the low adhesive areas is much larger than the size of a single domain with about $2 \mathrm{~nm}$ size as retrieved from TEM measurements [34]. In addition, the large low-adhesive areas often have a visible inner structure with a much smaller size in the range of a few nanometer. Therefore we assume that these low adhesive areas include numerous ionic domains. Since the water tends to form cluster inside the membrane it can be expected that smaller water-filled ionic areas coalesce to form larger waterrich interconnected ionic regions when they are closely neighbored. An example of an adhesion image at a cross section stored and measured at $50 \%$ rh is given in Figure 5a. Larger, partially connected low-adhesive regions are visible. In Figure 5b an adhesion measurement of a cross section cut from a sample that had been stored in water for 1 day after cutting is given. This image exhibits numerous water-filled regions with a clear inner-structure. In both samples, the water-filled area might not be at equilibrium state, where more circular structures are expected. An example of a cross section in equilibrium is given in Figure $5 \mathrm{c}$. The sample has been cut and then stored for 1 day over a saturated $\mathrm{NaCl}$-solution at $75 \%$ rh. It is most likely that in 
addition to the evaporation of water also $\mathrm{NaCl}$ molecules were evaporated. Their deposition on the sample may have increased the surface humidity, also indicated by the high adhesion values. Large, $200 \mathrm{~nm}$-wide circular areas with low adhesion and an inner structure are visible together with several much smaller, approximately 50nm-wide dark circular areas.

The typical circular shape under equilibrium conditions is probably induced by the surface tension of the water droplet. Both, the circular shape and the small size distribution indicate that at equilibrium, due to a size-dependent water pressure, where smaller particles/drops have a higher pressure, diffusion of water leads to a further growth of larger water-rich areas [14]. The smaller water-filled areas disappear with time, and for larger ones, the water molecules do not leave the droplet as easily. These large water-filled areas that include ionic domains are consistent with the model of Gebel, where fibrillar objects surrounded by water are proposed [16].

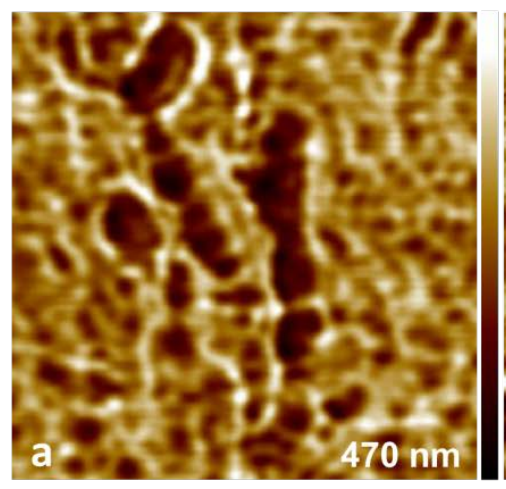

$\Delta \mathrm{F}=7.2 \mathrm{nN}$

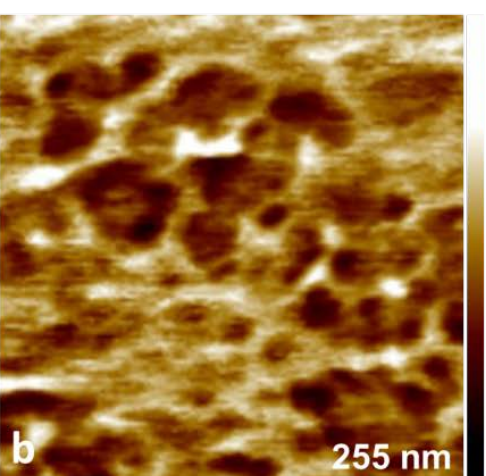

$\Delta \mathrm{F}=1.3 \mathrm{nN}$

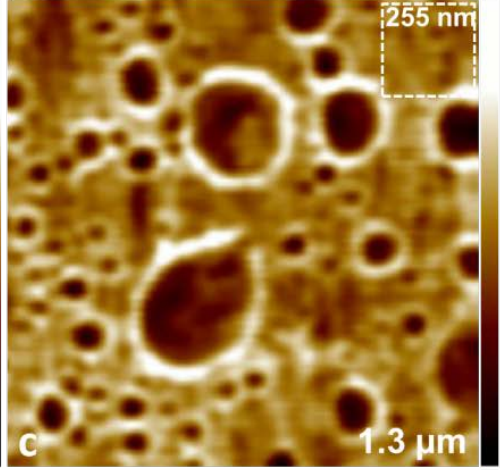

$\Delta \mathrm{F}=13 \mathrm{nN}$

Figure 5: Adhesion force images of cross sections of non-activated Nafion $® 212$, (a) equilibrated at 40-50 \% rh, measured at $50 \%$ rh; (b) equilibrated at $40-50 \%$ rh, cross section stored in water for 1 day, measured at $50 \%$ rh, (c) equilibrated at 40-50 \% rh, cross section stored for 1 day over saturated $\mathrm{NaCl}$-solution at $75 \%$ rh, measured at $30-40 \%$ rh.

The distribution of the water-rich ionic phase at the surface, as shown in Figure 6 for different ionomer samples, is visible in the capacitive current mapping even if the 
ionic phase is not connected to a continuous ionic network. Generally, a locally higher capacitive current (bright area in Figures 6a,b) indicates locally higher water content, and the distribution of water-rich and water-poor areas can be imaged [14].

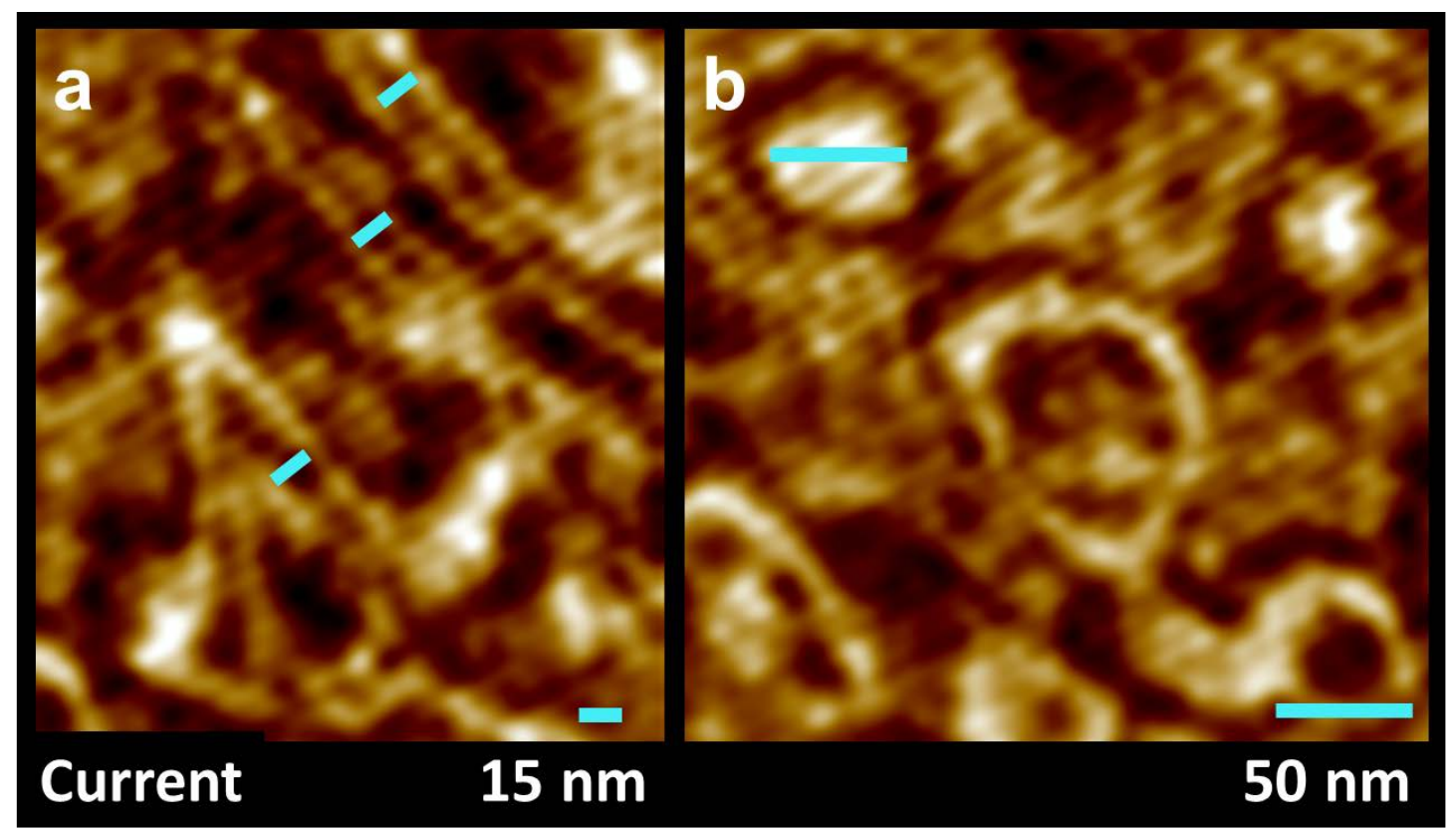

Figure 6: Adhesion image of cross sections of non-activated membrane samples of Nafion ${ }^{\circledR} 212$, equilibrated and measured at 30-40 \% rh: (a) area with layered water sheets, and (b) area with circular and ring-like water-filled layers.

The mapping of capacitive current at $\mathrm{Nafion}^{\circledR} 212$ with two distinct structures is shown in Figure 6. In comparison with Figure 2, where large lamellar structured areas are present, one would expect large regions with parallel water layers to be visible in the capacitive current images. In general, the formation of the two phases in the ionomer upon solidification can be regarded to be somewhat similar to the formation of a colloidal phase. Depending on the local surface energy and percentage of solvent, different structures are stable, including lamellar sheets, micelles, or inverted micelles [15],[44]. The reduced lamellar ordering in this case is most likely caused by increased water content. In this sample, the lamellar structures are visible only in a few regions. In Figure 6, two structurally different areas are shown. In Figure 6a, 
parallel oriented water-rich layers are present that resemble the lamellar structures in Figure 2. The layer thickness of the enclosed non-conductive layers is consistent with the 6-nm-wide lamellae derived from the step height measurements. Figure 6b shows an area with circular water inclusions of approximately 50-nm size. The water-filled layers are buckled caused by the clustering of sulfonic acid end groups, forming ringlike structures or larger areas. It also becomes clear that upon drying of the sample, a previously regular stacking cannot easily be restored again, and a continuous structure change must occur upon further change of the conditions.

\subsubsection{Non-equilibrium structure of ionomer}

Faradaic current was detected only after activation; storage in water was not sufficient. At freshly cut cross sections, large continuous but laterally heterogeneous current flow was detected across ionic areas of several tens of nanometers to 100 $\mathrm{nm}$ [14]. In contrast, at surfaces clustered but isolated current spots were detected [8]. In technical applications, such as fuel cells or electrolysis, faradaic current flow is always present.

In contrast to the equilibrium case, the formation of a water-filled interconnected ionically conducting phase by external activation is expected to result in anisotropic orientation of the water-rich ionically conducting phase. Due to mechanical water pressure associated to proton flow, a stretching of water-filled phase in the direction of the applied voltage can be expected. In Figure $7 \mathrm{a}$ and Figure $7 \mathrm{~b}$, topography and corresponding adhesion image of the cross section of a sample that was activated before cutting are presented. The direction of voltage application is given in Figure 7e. During microtome cutting, the sample was disrupted between two layers. At the surface in Figure 7a, in addition to numerous circular features, long, branched, elevated, channel-like structures are present with a height of $5 \mathrm{~nm}$. As determined 
from the height profile across one channel in Figure $7 d$, marked in the height image Figure 7c, typical diameters of approximately $50 \mathrm{~nm}$ and lengths up to the order of micrometers were observed. These areas exhibit low adhesion, as visible in Figure $7 \mathrm{~b}$, and therefore represent the water-filled interconnected ionically conductive phase. At certain positions, more extended circular structures with diameters up to a few hundred nanometers are observed and are connected by the longer channels. These channel-like structures are extremely flat. At a few positons, these structures stack on each other with 10-nm heights. It can be concluded that the observed stretching of this water-filled ionically conducting phase in the direction of the applied voltage directly represents the formation of a continuous ionic conducting network that was not present before current flow. It was concluded that the concurrent water flow with proton current, the electro-osmotic drag, facilitates the coalescence of isolated water-filled ionically conductive regions in the pressure direction, thereby filling the ionically conductive network with water. Subsequent proton flow increases the anisotropic changes of the internal ionically conductive phase structure and at the interface, where the conductive pores evolve [8]. Consequently, only a part of the ionic domains may participate in the continuous conducting network. 

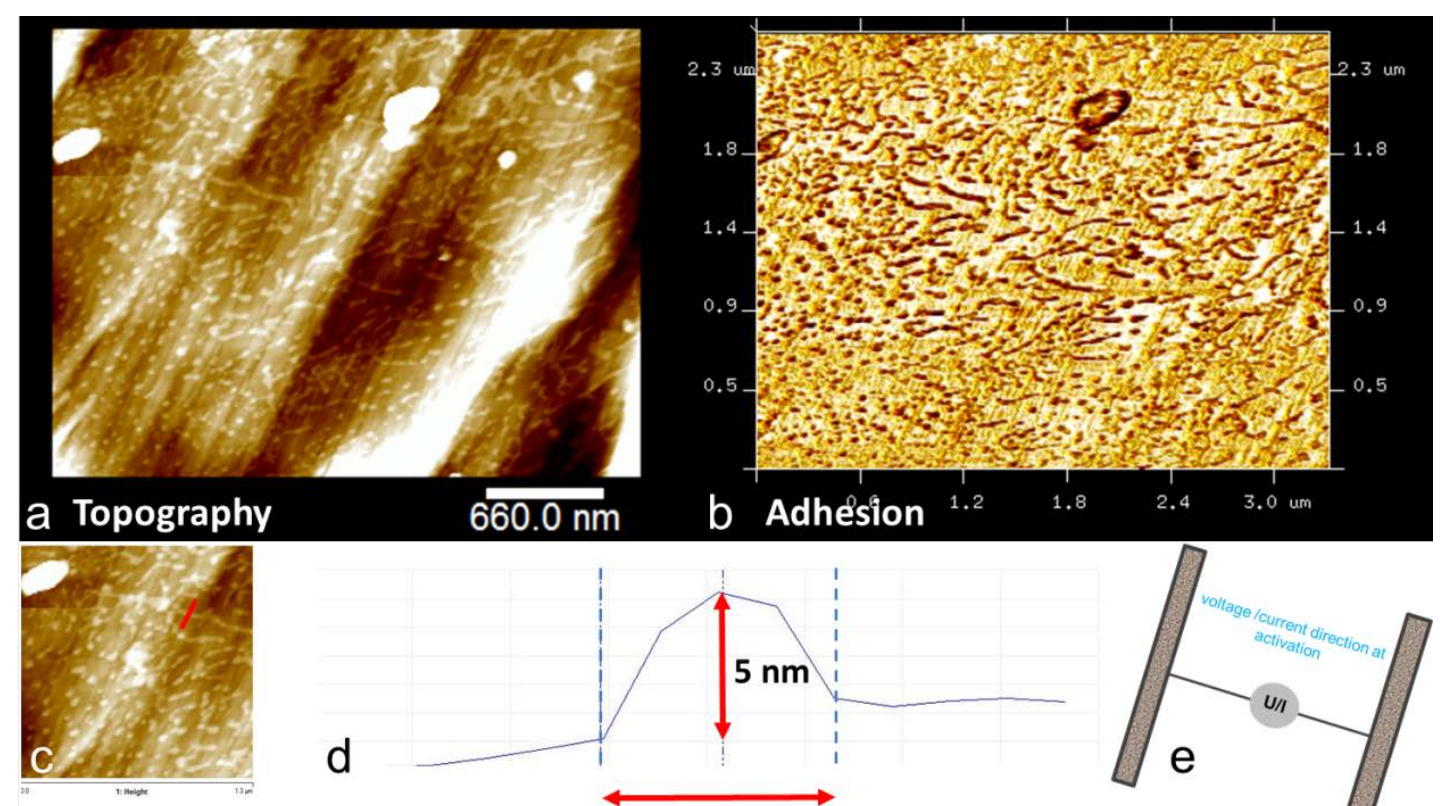

d
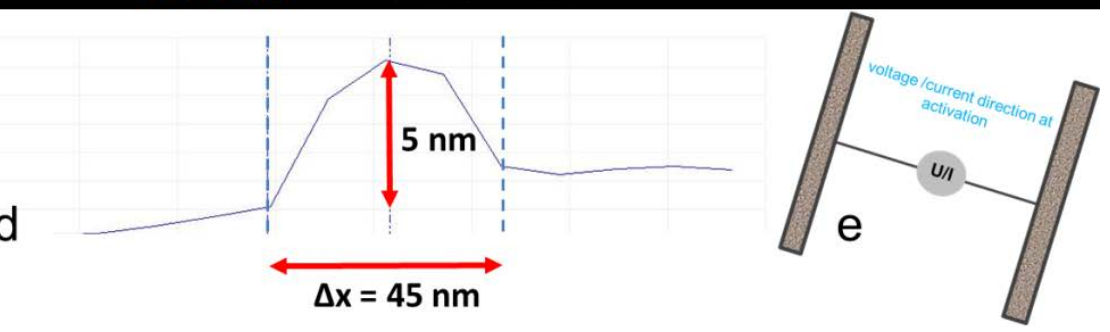

Figure 7: (a) 3D topography image of cross section of non-activated Nafion ${ }^{\circledR} 212$, equilibrated and measured at 30-40 \%, (b) adhesion force mapping overlaid on topography, (c) zoomed-in 3D topography image, (d) height profile along the marked line, and (e) direction of applied voltage for the activation procedure for all images.

\subsubsection{Platinum deposits in the ionomer membrane}

Platinum as a metal differs strongly in its properties from polymeric Nafion ${ }^{\circledR}$, both in terms of mechanical and electrical properties. In addition to its electronic conductivity, not present in the ionomer membrane, in AFM mappings bare platinum exhibits a much lower adhesion force compared with Nafion ${ }^{\circledR}$ [45]. Even when covered by a thin polymer layer, platinum particles can be detected by their higher stiffness as demonstrated in [45], but also by their lower surface potential. Therefore, in a compound, these two materials can be unambiguously discerned by a combination of conductive and material-sensitive AFM. 


\subsection{Interface formation of ionomer layer to hydrophobic and hydrophilic environment}

The formation of the ionomer interface structure that influences the interface conductivity, i.e., between membrane and the ionomer content of the electrode, and between the catalyst-coated carbon particles and the ionomer, was investigated. For studying the formation of hydrophobic and hydrophilic domains and their orientation toward the interface under dry and wet conditions, two ionomer layers were subsequently applied from ionomer dispersion. The interface of the first layer to ambient air was dried under low humidity conditions, and the interface of the second top layer was dried in neighborhood to the wet ionomer. An adhesion image of a cross section through the interface is given in Figure 8. The first applied ionomer layer that was dried in ambient air with a relative humidity of approximately $30-40 \%$ is visible at the left side. The violet color indicates a high adhesion force, and green indicates lower adhesion values. In Figure 8b, the formation of differently adhesive structures, hydrophobic/PTFE-rich and hydrophilic/water-rich, both orientated parallel to the interface, is visible. Higher adhesion values typically indicates a higher PTFElike content [46], which in this case, is associated with a backbone-rich phase; low adhesion indicates a water-rich phase, as discussed above. Close to the interface, more of the higher adhesive water-poor phase is present, which agrees with measurements at membranes [21]. The total thickness of this layer, which is oriented parallel to the interface, is approximately $2 \mu \mathrm{m}$. This thickness is much larger than that reported in the literature [21], [19]; however, these layers were quite fresh and were not heated above the glass transition temperature. Under the common procedures used for fuel cells, the layers will most likely dry out further and shrink with time. On the right side of the interface, the second applied ionomer part can be discerned. This component was applied after drying of the first layer at a relative 
humidity of $30-40 \%$ for one day. The vertical orientation of i.e., the green ionomer phases with lower adhesion to the interface is clearly visible. In addition, on average this second layer has an overall lower adhesion. These results support the expectation for interface formation in a wet environment without skin layer formation. The equilibrium structure of the interfaces results from the balance of the different surface energies. The equilibrium structure in air / water-vapor is characterized by a hydrophobic water-poor surface skin layer with highly crystalline polymer structures oriented roughly parallel to the surface, as observed in Figure 8a [21],[22]. The existence of hydrophobic structures at a membrane surface and their resulting lack of conductivity were measured by AFM in a previous study [8]. For an aqueous liquid environment, the formation of polymer bundles oriented vertically to the interface is expected as shown in Figure 8d [22]. In conclusion, we expect that from the orientation of hydrophobic/hydrophilic domains in Nafion particles or films inside the electrode towards the interface with Pt/carbon, the surface properties of the interface can be derived.

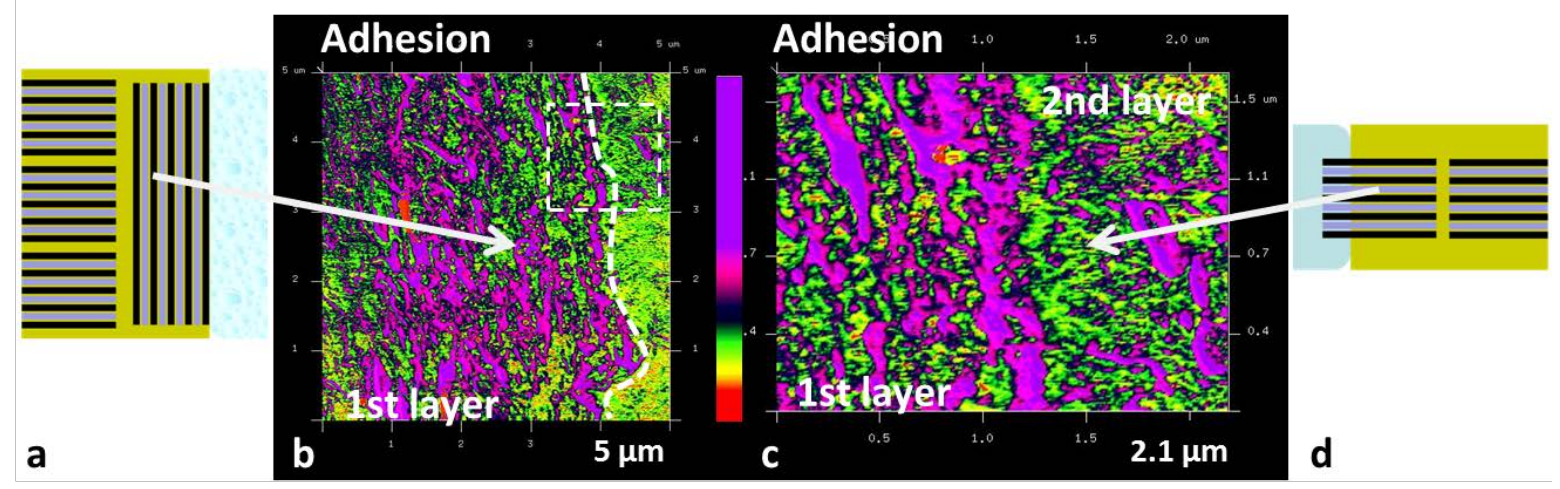

Figure 8: Adhesion images of a cross section of non-activated sample prepared from two layers of ionomer dispersion after drying for one day at 30-40 \% rh, measured at 30-40 \% rh: (a) scheme of orientation of polymer structures in hydrophobic environment, (b) adhesion mapping of the interface region, (c) zoomed-in view of the marked area of the adhesion image in (b), and (d) scheme of orientation of polymer structures in hydrophilic environment. 


\subsection{AFM investigation of cells}

In this section, the application of material-sensitive and conductive AFM to the investigation of cross sections of fuel cell components is demonstrated. Slices of MEAs with an enclosed reinforced ionomer layer and embraced electrodes were investigated. It will be demonstrated that AFM can be used to analyze the ionomer conductivity of the membrane and electrodes after MEA fabrication and can identify the ionomer content inside the electrodes.

\subsubsection{Membrane electrode assembly}

The analyzed AQUIVION ${ }^{\circledR}$ PFSA-based MEA cross section images with $30-\mu \mathrm{m}$ side lengths are presented in Figure 9. The non-operated and non-activated sample was equilibrated and measured at 30-40 \% rh. At the left, the topography (Figure 9a) reveals a difference in the height after microtome cutting, which reflects the different mechanical properties of the two electrodes at both sides and the membrane. The neighboring elevated swollen ionomer layers sandwich the reinforcement layer with lower height at the center. The different stiffness values of these components, with the highest values at the electrodes and a higher stiffness at the reinforced ionomer compared with the pure ionomer are recognizable in Figure 9b. The electronic current flow at a voltage of $U=100 \mathrm{mV}$, applied between the conductive AFM tip and the conductive back contact of the entire cross section, is restricted to the electrodes (Figure 9c). Their conductivity is laterally inhomogeneous. The applied voltage was too low to induce ionic current flow through the ionomer. On this scale, no phase separation is visible in the ionomer. 


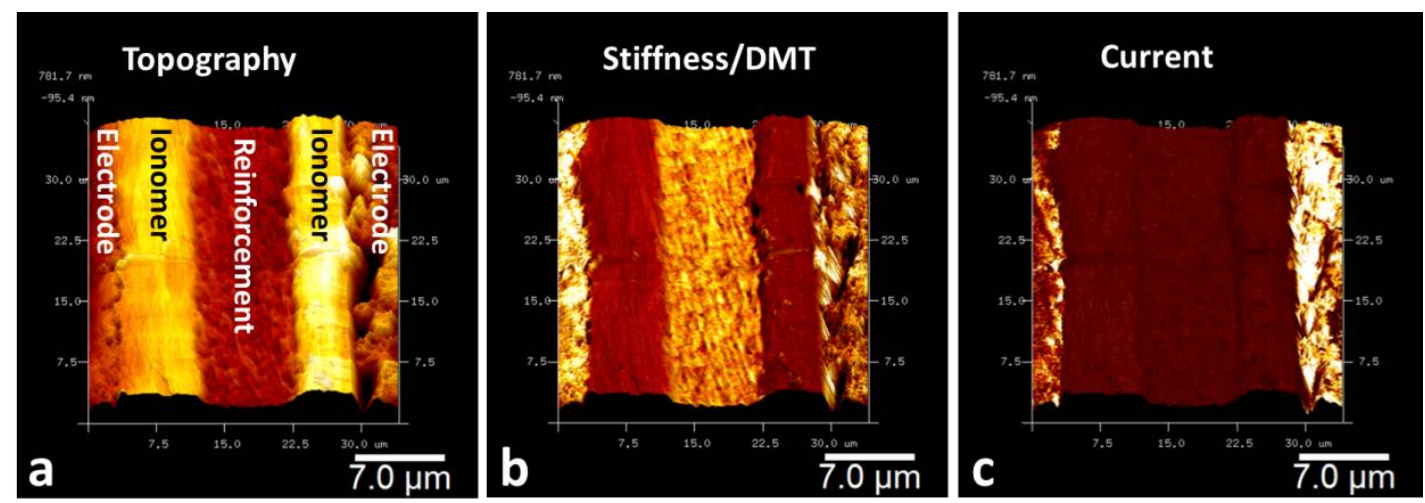

Figure 9: Cross section of a pristine MEA with AQUIVION ${ }^{\circledR}$ PFSA membrane, equilibrated and measured at 30-40 \% rh: (a) 3D topography image, (b) stiffness overlaid on 3D topography, and (c) current values overlaid on topography.

In Figure 10, the electronic and the ionic conductivity were measured at the cathode side of a pristine Nafion ${ }^{\circledR}$-based MEA sample on an area that comprises only one half of the MEA. The MEA was equilibrated at 30-40 \% rh and measured at the same humidity in air. The adhesion values of the electrode, ionomer, and reinforced membrane also differ (Figure 10a). As expected from the component characterization, the platinum/carbon-rich electrode exhibits a lower adhesion, and the reinforced PTFE-rich part exhibits a higher adhesion force compared with the pure ionomer. Figures $10 \mathrm{~b}$ and $\mathrm{c}$ show the same measurement but with different current magnification. In Figure 10b, the inhomogeneous current distribution of the electrode with large non-conductive inclusions is clearly visible. In Figure 10c, the current was further magnified to expose the small capacitive conductivity of the ionomer. Differently conductive stripes oriented parallel to the electrode interface are visible, which represent the water-rich layers. In this large-area scan, the smallest conductive layers were 100-nm thick. This sample was not operated or otherwise activated after MEA preparation or before AFM analysis. Therefore, the parallel water-filled layers indicate the existence of an ordered lamellar ionomer structure that formed after hot pressing and subsequent cooling of the ionomer, similar to the lamellar ordered membranes cross section presented in Figure 2. No conductivity 
can be observed beyond the interface of the reinforcement layer at the right. The ionomer content in the reinforcement in this pristine state at low humidity was obviously too small to be detected.
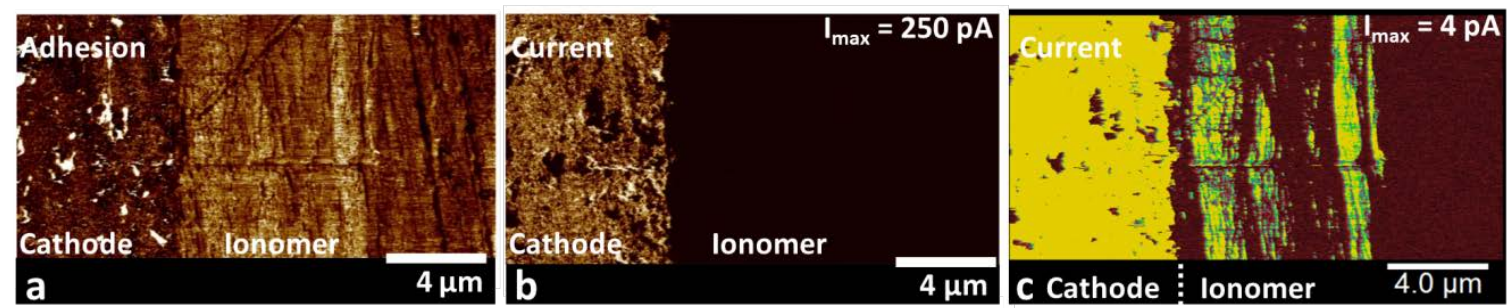

Figure 10: Cross section of one half of a pristine Nafion ${ }^{\circledR}$-based MEA, equilibrated and measured at 30-40 \% rh, sample wetted before measurement: (a) adhesion mapping, (b) current values at 2-V bias voltage, and (c) current values from (b) with a magnified current scale.

\subsubsection{Catalytic layer}

Fuel cell electrodes typically consist of approximately 30 wt.\% ionomer mixed with platinum-catalyst-covered mesoporous carbon to form a porous, electronically and ionically conducting layer that permits gas, water, and current transport. The interface of platinum catalyst particles with the ionomer can be compared with a wet environment, considering that platinum is always covered with water [47]. The surface of the mesoporous carbon is assumed to be partly hydrophilic mixed with hydrophobic areas. Therefore, for ionomer particles next to platinum/carbon, the formation of a vertically oriented ionic/backbone phase is expected, provided that the ionomer phase is sufficiently large to develop the typical phase separation. According to experiments with thin $\mathrm{Nafion}^{\circledR}$ layers, membrane properties change below a thickness of approximately $50 \mathrm{~nm}$ [48]. Ultra-thin Nafion ${ }^{\circledR}$ layers that have selfassembled on platinum or silicon have a minimum thickness of $4 \mathrm{~nm}$ in air [49]. The minimum layer thickness of the ionomer layer around platinum-catalyst-covered carbon particles could therefore be expected between particles in fuel cell electrodes 
[50]. Molecular dynamic calculations predict a layer thickness of $4.5 \mathrm{~nm}$ under wet conditions between a hydrophilic and a hydrophilic border, including a water layer [50].

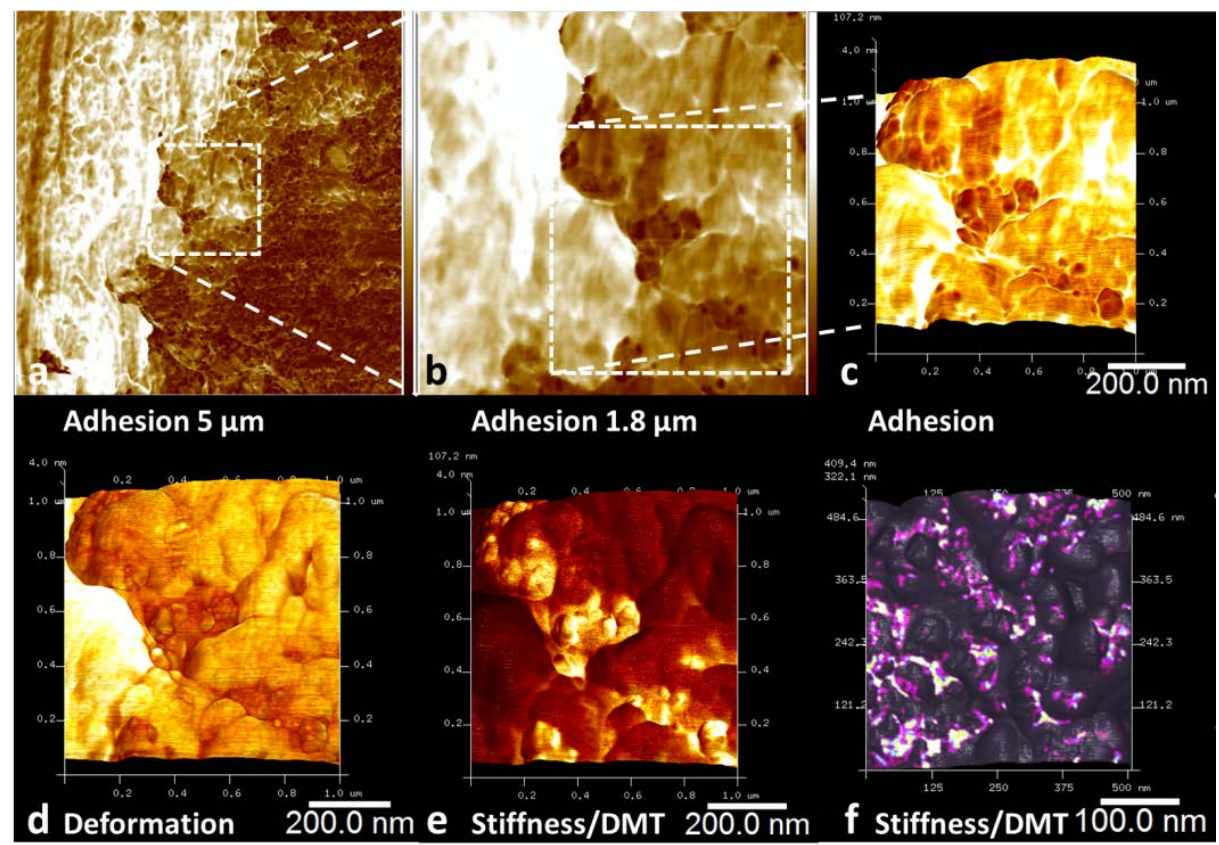

Figure 11: Cross section of the membrane-electrode interface of a pristine Nafion ${ }^{\circledR}$ based MEA, equilibrated and measured at 30-40 \% rh: (a) adhesion mapping, (b) zoomed-in adhesion mapping of region marked in (a), (c) adhesion mapping of zoomed-in area marked in (b) overlaid on 3D topography, (d) deformation mapping overlaid on 3D topography, (e) stiffness mapping overlaid on 3D topography, and (f) stiffness mapping of an electrode overlaid on 3D topography.

The interface between the ionomer layer and the electrode can be observed in Figures 11 a to e. In the adhesion force, the bright, high-adhesive ionomer can be distinguished from the dark, low-adhesive carbon/Pt-rich phase. The interface is clearly visible and well defined but not straight on this scale. In the higher magnified image of Figure 11b, darker carbon/Pt-rich particles can be clearly discerned. Inside some of these particles, very dark (low-adhesive) agglomerated Pt-rich inclusions appear. The bottom part of the interface (marked in Figure 11b) is magnified as a 3D topography image overlaid with adhesion in Figure 11c, as deformation mapping in Figure 11d, and as stiffness image in Figure 11e. A clear separation between the 
$\mathrm{Pt} / \mathrm{C}-$ rich particles and the ionomer is visible. $\mathrm{Pt} / \mathrm{C}$ is discerned by its lower adhesion (Figure 11c) and lower deformation, in the latter image with lower lateral resolution due to a larger contact area at impression. A clear difference exists between the soft ionomer with low stiffness (the dark color in Figure 11e) and the high stiffness of $\mathrm{Pt} / \mathrm{C}$ component. A significant difference in the stiffness between metallic platinum and carbon, with the latter being less stiff, is expected and leads to an internal structure of the overall brighter particles. It can be assumed that the brightest spots indicate the positions of platinum particles or agglomerates on the surface. The imaging of single 4-nm-sized Pt-particles on 30-nm-wide carbon is presented in a higher magnified stiffness mapping (Figure 11f), where carbon and metallic platinum differ significantly. However, for a quantitative analysis of the Pt-content, the uncertainty in the resulting particle number may be too large due to the possible loss of platinum induced by the cutting process.

The application of ionomer detection is further demonstrated in Figure 12. The surface of the $1-\mu \mathrm{m}^{2}$ large cut is quite smooth, as observed in the 3D topography image in Figure 12a. No significant difference is visible between the ionomer particles and $\mathrm{Pt} / \mathrm{C}$ content. To discern the ionomer content from the $\mathrm{Pt} / \mathrm{C}$ particles, the electronic conductivity (Figure 12b) and the adhesion mapping (Figure 12c) were compared. In the current image, large non-electronically conductive parts, partly marked by dotted lines, are present. These components cover an area of approximately $12 \%$ of the imaged area. Confirmation that these non-conductive areas consist of ionomer is obtained from the adhesion mapping. Ionomer particles appear as bright parts at identical positions as the non-conductive areas, as visible in the dotted markings. 


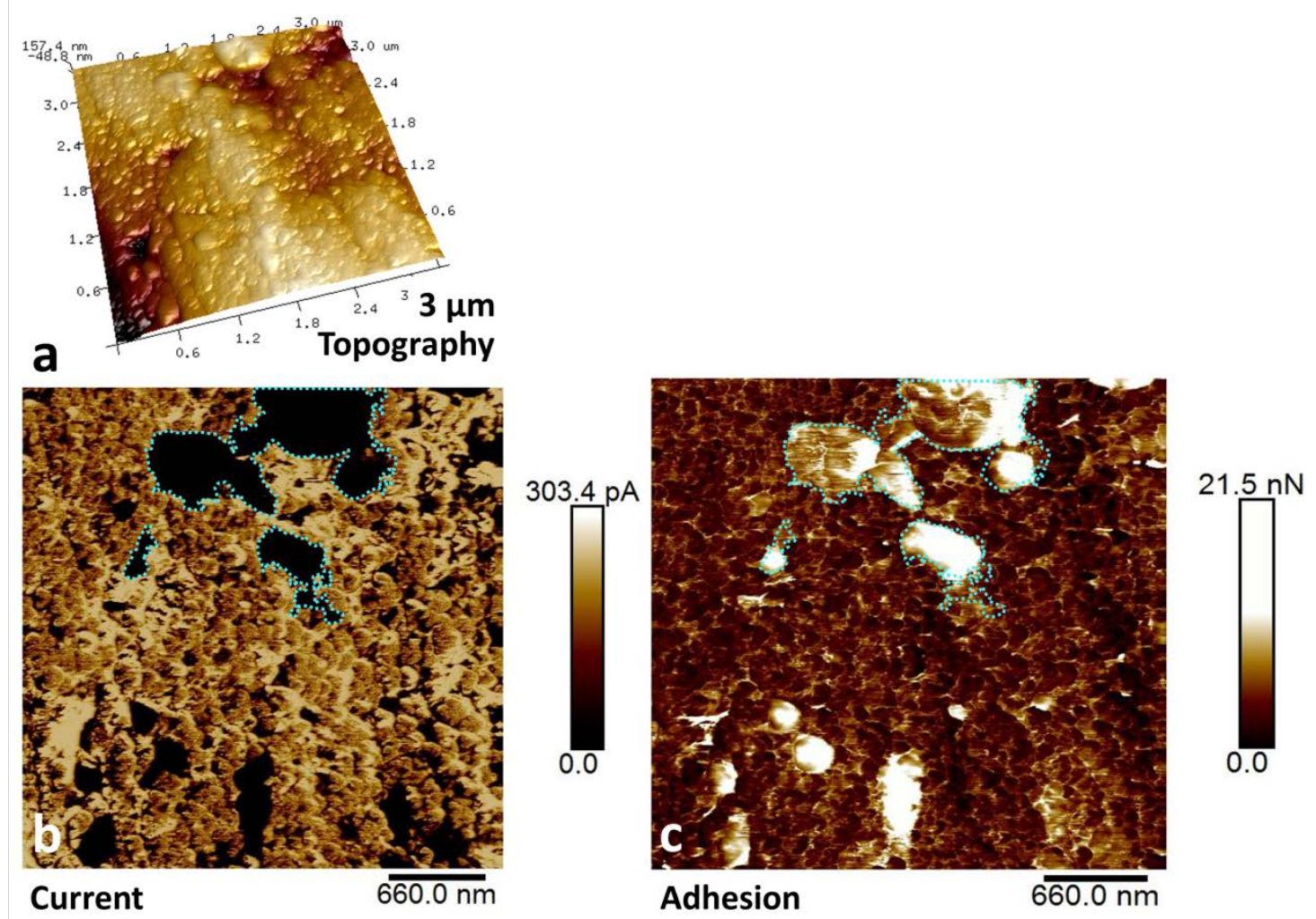

Figure 12: Cross section of the electrode of a pristine Nafion ${ }^{\circledR}$-based MEA, equilibrated and measured at 30-40 \% rh: (a) 3D topography image, (b) current mapping at bias voltage $\mathrm{U}=2 \mathrm{~V}$, and (c) adhesion mapping.

The identification of the ionomer content in the electrode was used to detect a gradient in the ionomer content from the electrode interface across the entire cathode. In Figure 13, a diagram of the average ionomer content of an area of $1 \mu \mathrm{m}^{2}$ calculated from the adhesion images, represented by the white dots and measured across the electrode with a step width of $1 \mu \mathrm{m}$, is overlaid on its adhesion image. The ionomer content decreases with increasing distance from the membrane-electrode interface. The higher ionomer content at the membrane-electrode interface may be caused by the hot-pressing step of the MEA fabrication. 


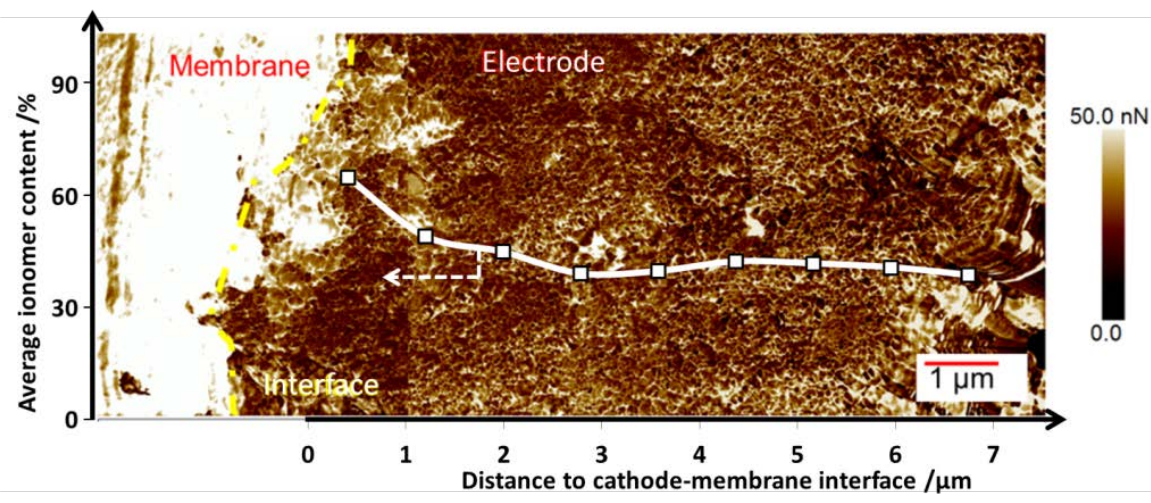

Figure 13: Adhesion image of a cross section of the catalytic layer of a pristine Nafion ${ }^{\circledR}$-based MEA, equilibrated and measured at 30-40 \% rh, overlaid with a diagram where white dots indicate the average ionomer content of an area of $1 \mu \mathrm{m}^{2}$ with 1- $\mu \mathrm{m}$ step width.

The layer thickness near Pt/C particles in the electrode can be small, and their identification requires a high resolution and a sufficiently sharp AFM tip. In addition, the gap between two particles may lead to an increase of the adhesion force induced by the tip contact area increase due to geometric considerations. This effect cannot be distinguished from the high adhesion of the ionomer. To avoid this artifact of the measurement, the thickness of small layers with increased adhesion was measured at a flat interface between two particles. The topography of the catalytic layer is shown in Figure 14a, and the corresponding adhesion mapping is presented in Figure 14b. Figure 14c shows a flat area in the topography image; the adhesion profile (Figure 14e) and height profile (Figure 14f) were measured along the marked lines. The first high adhesive peaks at the left side in the profile line positioned between two particles correspond to flat regions, as visible in the height profile. The adhesion peak on the right side corresponds to a gap, where the adhesion value may be increased by an increased contact area. For the first two structures the high adhesion structure can be unambiguously attributed to an ionomer layer. The layer thickness evaluated this way cannot be measured precisely, for the smallest layers a value in the order of roughly 4-7 $\mathrm{nm}$ could be estimated, but it is not statistically 
representative; an extensive statistical investigation of larger areas is required which is in preparation. A minimum layer thickness of $4 \mathrm{~nm}$ has been reported for selfassembled ultra-thin Nafion ${ }^{\circledR}$ layers [48]. Following the model calculation of Borges et al. [51] for ultra-thin Nafion ${ }^{\circledR}$ layers in contact with a hydrophilic surface where a similar thickness could be derived, the formation of a water layer, and therefore proton conductivity, would then be expected along the surface. It also seems that a high percentage of the Pt/C particles are covered by ionomer, which could generate a high gas diffusion barrier.

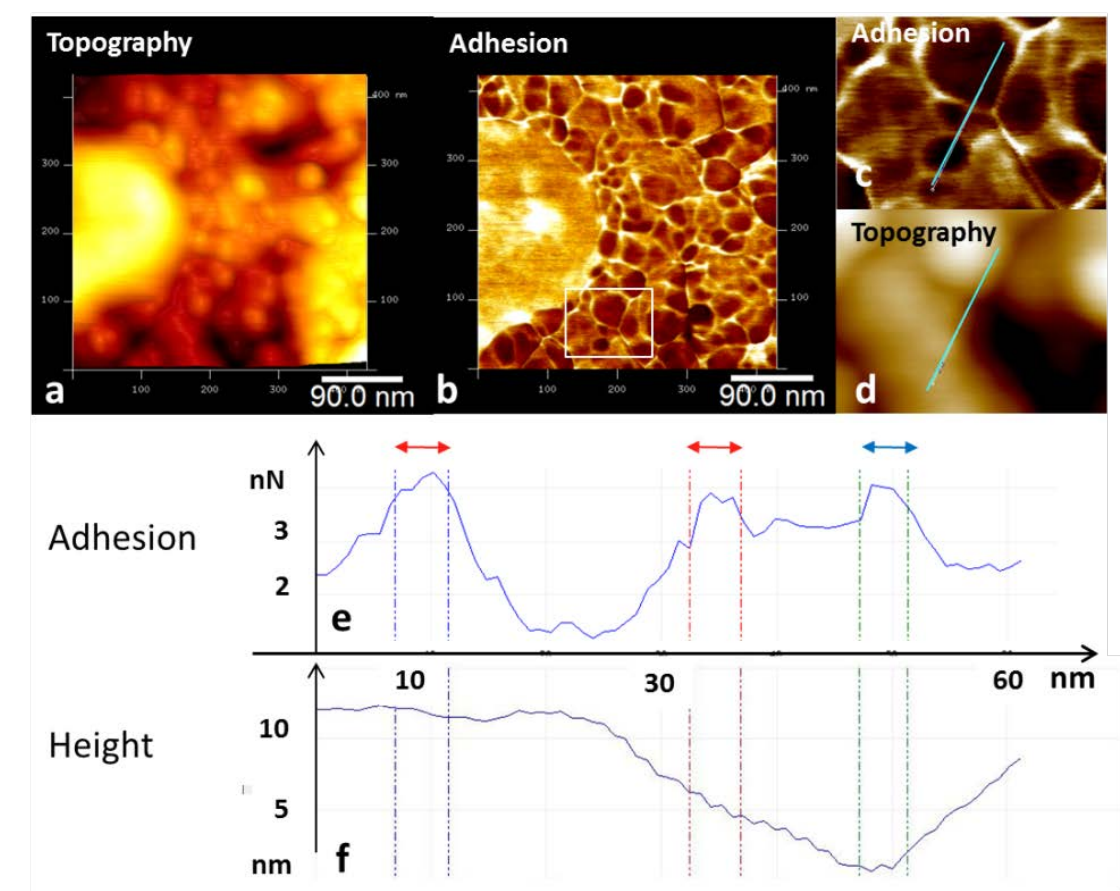

Figure 2: Cross section of the electrode of a pristine Nafion ${ }^{\circledR}$-based MEA equilibrated and measured at 30-40 \% rh: (a) 3D topography, (b) adhesion mapping, (c) adhesion image of zoomed-in area marked in (b), (d) topography image of zoomed-in area marked in (b), (e) adhesion profile along line marked in (c), and (f) height profile along the line marked in (d).

For the large ionic particles inside the electrode with diameters of approximately 500 $\mathrm{nm}$, visible in Figure 12, phase separation in these particles is expected. At a bias voltage of $2 \mathrm{~V}$, ionic current was detected at one of these large ionomer particles, as shown in Figure 15. The white parts of the current images represent electronically conductive $\mathrm{Pt} / \mathrm{C}$ particles that appear white because of the high current saturation 
values of $10 \mathrm{nA}$. Inside the ionomer, a current can be measured at this previously non-activated sample after scanning with the AFM tip at $2 \mathrm{~V}$. With a magnitude of 1.5 $\mathrm{nA}$, this current is most-likely a faradaic current. The difference in magnitude of the currents is better visible in the 3D-current view of Figure 15b. The brighter, waterfilled ionically conductive areas of the ionomer particles bridge the neighboring $\mathrm{Pt} / \mathrm{C}$ particles and permit ionic current flow between $\mathrm{Pt} / \mathrm{C}$ particles in the electrode. These conductive connections between catalyst particles are oriented vertically to the Pt/C interface, similar to the hydrophilic low-adhesive phase in Figure 8, formed at a wet ionomer membrane interface. Therefore, we conclude that the ionomer/Pt/C interface was hydrophilic in this case.
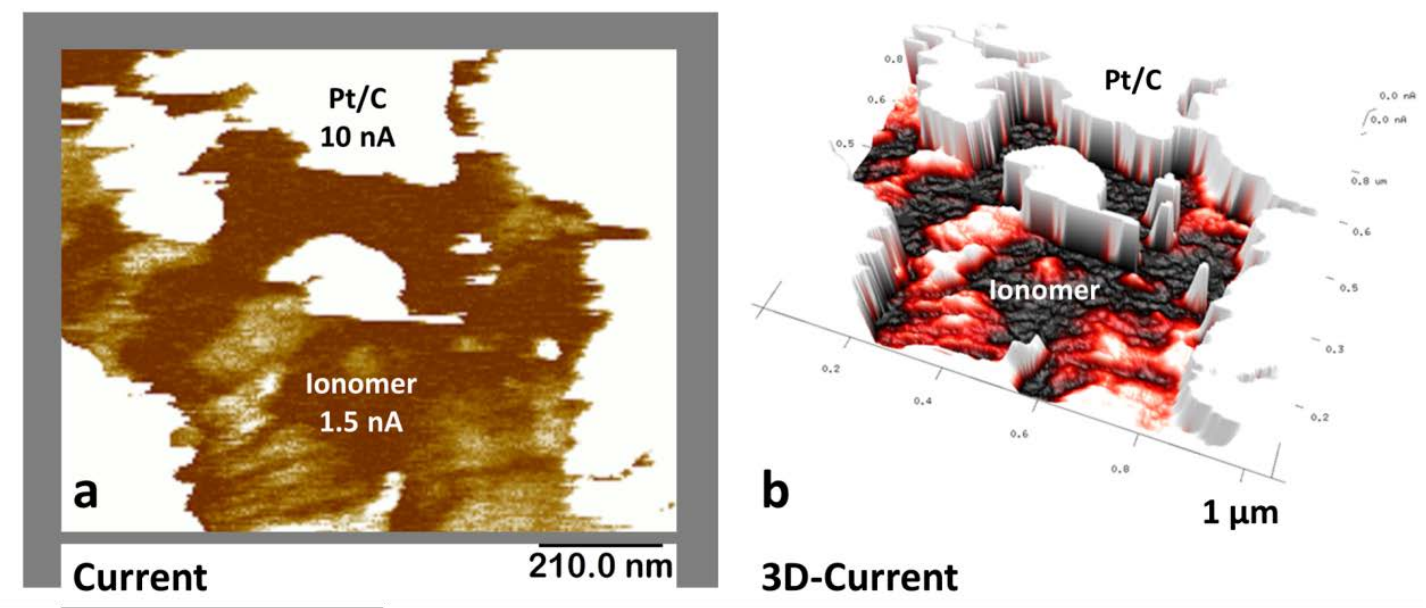

Figure 15: Cross section of an ionomer particle inside an electrode of a pristine Nafion $^{\circledR}$-based MEA, equilibrated and measured at 30-40 \% rh: (a) current mapping at $\mathrm{U}=2 \mathrm{~V}$ with high current magnification, and (b) 3D-view on current mapping of (a).

\section{Conclusions}

The combination of material-sensitive tapping mode AFM and conductivity measurements was used to identify and analyze different components and phases in fuel cell components and technical MEAs, both the membranes and catalytic layers. The hydrophilic/hydrophobic phase distribution of Nafion ${ }^{\circledR}$ membranes was analyzed. Water-filled interconnected ionically conductive regions could be recognized by their 
apparently low adhesion signal, which results from the local formation of a double layer in the ionomer. The large low-adhesive areas were explained by larger waterfilled regions that include several smaller ionic domains. The even distribution and circular shape (spherical in the volume) under conditions close to equilibrium were explained by the size-dependent water pressure of particles that leads to a growth of larger water drops. The circular (spherical in the volume) shape of this water-filled phase minimizes the interface energy.

After forcing a current flow through the sample a current-induced anisotropic orientation of water-filled sheets, also identified by their low adhesion, was observed. In the direction of current flow the formation of flat, micrometer-long, branched or circular, connected hydrophilic regions with a thickness of $5 \mathrm{~nm}$ and a width of 50-80 $\mathrm{nm}$ was observed. The forced-current flow led to the formation of a water-filled continuous network, which could be directly observed by AFM. It was concluded that such a continuous ionic network does not necessarily exist in pristine membranes, because before forced current flow the low-adhesive areas were isolated. The presence of large high-adhesive backbone-rich regions with an ordered lamellar structure was observed. Measurements of the layer thickness confirmed the existence of polymer crystalline lamellae with a spherulitic structure and a thickness of roughly $6 \mathrm{~nm}$. The smaller steps heights were also consistent with a spherulitic structure and zig-zag-folded polymer main chains containing the solvated ionic side groups in between.

It was demonstrated that with material-sensitive AFM the identification of the ionomer content inside the electrode could be used to monitor the relative ionomer distribution across an electrode, particles and films. Higher ionomer content was observed close to the electrode interface. The distribution of ionomer particles inside the catalytic layers was inhomogeneous with large ionomer particles. Detection of cross current 
inside one of those particles was performed, and the orientation of the ionically conductive phase indicated a hydrophilic interface to Pt/C.

\section{Acknowledgements}

The research leading to these results has received funding from the European Union's Seventh Framework Programme (FP7/ 2007-2013) for the Fuel Cells and Hydrogen Joint Technology Initiative under grant agreement $n^{\circ} 303452$, “IMPACT_Improved Lifetime of Automotive Application Fuel Cells with ultra-low Pt-loading". The research leading to these results has received funding from the European Union's Seventh Framework Programme (FP7/ 2007-2013) for the Fuel Cells and Hydrogen Joint Technology Initiative under grant agreement n³03452, "IMPACT— Improved Lifetime of Automotive Application Fuel Cells with ultra-low Pt-loading". We gratefully acknowledge the supply of aged half-MEA-samples by Xiao-Zi Yuan and Haijiang Wang, National Research Council Canada, Vancouver, BC.

\section{References}

[1] K.A. Mauritz, R.B. Moore, State of understanding of Nafion, Chem. Rev. 104 (2004) 4535-4586.

[2] S. Subianto, M. Pica, M. Casciola, P. Cojocaru, L. Merlo, G. Hards, et al., Physical and chemical modification routes leading to improved mechanical properties of perfluorosulfonic acid membranes for PEM fuel cells, J. Power Sources. 233 (2013) 216-230.

[3] Melchy, P. É. A., Eikerling, M., Physical theory of ionomer aggregation in water, Meet. Abstr. Electrochem. Soc. 15 (2013) 1391-1391.

[4] M. Ludvigsson, J. Lindgren, J. Tegenfeldt, Crystallinity in cast Nafion, J. Electrochem. Soc. 147 (2000) 1303-1305.

[5] K.-D. Kreuer, G. Portale, A Critical Revision of the Nano-Morphology of Proton Conducting Ionomers and Polyelectrolytes for Fuel Cell Applications, Adv. Funct. Mater. 23 (2013) 5390-5397.

[6] G. Alberti, R. Narducci, M. Sganappa, Effects of hydrothermal/thermal treatments on the water-uptake of Nafion membranes and relations with changes of conformation, counter-elastic force and tensile modulus of the matrix, J. Power Sources. 178 (2008) 575-583. 
[7] M. Adachi, T. Navessin, Z. Xie, B. Frisken, S. Holdcroft, Correlation of In Situ and Ex Situ Measurements of Water Permeation Through Nafion NRE211 Proton Exchange Membranes, J. Electrochem. Soc. 156 (2009) B782.

[8] R. Hiesgen, S. Helmly, I. Galm, T. Morawietz, M. Handl, K.A. Friedrich, Microscopic Analysis of Current and Mechanical Properties of Nafion ${ }^{\circledR}$ Studied by Atomic Force Microscopy, Membranes. 2 (2012) 783-803.

[9] T.D. Gierke, G.E. Munn, Fc. Wilson, The morphology in nafion perfluorinated membrane products, as determined by wide-and small-angle $\mathrm{x}$-ray studies, $\mathrm{J}$. Polym. Sci. Polym. Phys. Ed. 19 (1981) 1687-1704.

[10] A. Eisenberg, Clustering of ions in organic polymers. A theoretical approach, Macromolecules. 3 (1970) 147-154.

[11] M. Falk, An infrared study of water in perfluorosulfonate (Nafion) membranes, Can. J. Chem. 58 (1980) 1495-1501.

[12] M. H. Litt, Polym Prepr. 38 (1997) 40.

[13] H.-G. Haubold, T. Vad, H. Jungbluth, P. Hiller, Nano structure of NAFION: a SAXS study, Electrochimica Acta. 46 (2001) 1559-1563.

[14] R. Hiesgen, T. Morawietz, M. Handl, M. Corasaniti, K.A. Friedrich, Insight into the Structure and Nanoscale Conductivity of Fluorinated Ionomer Membranes, J. Electrochem. Soc. 161 (2014) F1214-F1223.

[15] G. Gebel, Structural evolution of water swollen perfluorosulfonated ionomers from dry membrane to solution, Polymer. 41 (2000) 5829-5838.

[16] L. Rubatat, G. Gebel, O. Diat, Fibrillar Structure of Nafion: Matching Fourier and Real Space Studies of Corresponding Films and Solutions, Macromolecules. 37 (2004) 7772-7783.

[17] L. Rubatat, A.L. Rollet, G. Gebel, O. Diat, Evidence of Elongated Polymeric Aggregates in Nafion, Macromolecules. 35 (2002) 4050-4055. doi:10.1021/ma011578b.

[18] J.A. Elliott, D. Wu, S.J. Paddison, R.B. Moore, A unified morphological description of Nafion membranes from SAXS and mesoscale simulations, Soft Matter. 7 (2011) 6820-6827.

[19] K.-D. Kreuer, The role internal pressure for the hydration and transport properties of ionomers and polyelectrolytes, Solid State Ion. 252 (2013) 93-101.

[20] M.J. Cheah, I.G. Kevrekidis, J. Benziger, Effect of Interfacial Water Transport Resistance on Coupled Proton and Water Transport Across Nafion, J. Phys. Chem. B. 115 (2011) 10239-10250.

[21] J. Tang, W. Yuan, J. Zhang, H. Li, Y. Zhang, Evidence for a crystallite-rich skin on perfluorosulfonate ionomer membranes, RSC Adv. 3 (2013) 8947-8952.

[22] M. Bass, A. Berman, A. Singh, O. Konovalov, V. Freger, Surface structure of Nafion in vapor and liquid, J. Phys. Chem. B. 114 (2010) 3784-3790.

[23] R. Hiesgen, S. Helmly, T. Morawietz, X.-Z. Yuan, H. Wang, K.A. Friedrich, Atomic force microscopy studies of conductive nanostructures in solid polymer electrolytes, Electrochimica Acta. 110 (2013) 292-305.

[24] A. Kusoglu, M.A. Modestino, A. Hexemer, R.A. Segalman, A.Z. Weber, Subsecond Morphological Changes in Nafion during Water Uptake Detected by Small-Angle X-ray Scattering, ACS Macro Lett. 1 (2011) 33-36.

[25] D.A. Bussian, J.R. O'Dea, H. Metiu, S.K. Buratto, Nanoscale current imaging of the conducting channels in proton exchange membrane fuel cells, Nano Lett. 7 (2007) 227-232.

[26] J.R. O'Dea, N.J. Economou, S.K. Buratto, Surface Morphology of Nafion at Hydrated and Dehydrated Conditions, Macromolecules. 46 (2013) 2267-2274. 
[27] M.A. Modestino, A. Kusoglu, A. Hexemer, A.Z. Weber, R.A. Segalman, Controlling Nafion structure and properties via wetting interactions, Macromolecules. 45 (2012) 4681-4688.

[28] M.A. Modestino, D.K. Paul, S. Dishari, S.A. Petrina, F.I. Allen, M.A. Hickner, et al., Self-assembly and transport limitations in confined Nafion films, Macromolecules. 46 (2013) 867-873.

[29] E. Aleksandrova, R. Hiesgen, K.A. Friedrich, E. Roduner, Electrochemical atomic force microscopy study of proton conductivity in a Nafion membrane, Phys. Chem. Chem. Phys. 9 (2007) 2735-2743.

[30] E. Aleksandrova, S. Hink, R. Hiesgen, E. Roduner, Spatial distribution and dynamics of proton conductivity in fuel cell membranes: potential and limitations of electrochemical atomic force microscopy measurements, J. Phys. Condens. Matter. 23 (2011) 234109.

[31] D.G. Sanchez, D.G. Diaz, R. Hiesgen, I. Wehl, K.A. Friedrich, Oscillations of PEM fuel cells at low cathode humidification, J. Electroanal. Chem. 649 (2010) 219-231.

[32] R. Hiesgen, E. Aleksandrova, G. Meichsner, I. Wehl, E. Roduner, K.A. Friedrich, High-resolution imaging of ion conductivity of Nafion ${ }^{\circledR}$ membranes with electrochemical atomic force microscopy, Electrochimica Acta. 55 (2009) 423-429.

[33] D. Susac, V. Berejnov, A.P. Hitchcock, J. Stumper, STXM Characterization of PEM Fuel Cell Catalyst Layers, ECS Trans. 50 (2013) 405-413.

[34] C. Wang, V. Krishnan, D. Wu, R. Bledsoe, S.J. Paddison, G. Duscher, Evaluation of the microstructure of dry and hydrated perfluorosulfonic acid ionomers: microscopy and simulations, J. Mater. Chem. A. 1 (2012) 938-944.

[35] R. Maoz, S.R. Cohen, J. Sagiv, Nanoelectrochemical Patterning of Monolayer Surfaces: Toward Spatially Defined Self-Assembly of Nanostructures, Adv. Mater. 11 (1999) 55-61.

[36] S. Holdcroft, Fuel Cell Catalyst Layers: A Polymer Science Perspective, Chem. Mater. 26 (2014) 381-393.

[37] S. Ma, C.-H. Solterbeck, M. Odgaard, E. Skou, Microscopy studies on proton exchange membrane fuel cell electrodes with different ionomer contents, Appl. Phys. A. 96 (2009) 581-589.

[38] D. Susac, V. Berejnov, A.P. Hitchcock, J. Stumper, STXM Study of the Ionomer Distribution in the PEM Fuel Cell Catalyst Layers, ECS Trans. 41 (2011) 629635.

[39] V. Berejnov, D. Susac, J. Stumper, A.P. Hitchcock, 3D Chemical Mapping of PEM Fuel Cell Cathodes by Scanning Transmission Soft X-ray SpectroTomography, ECS Trans. 50 (2013) 361-368.

[40] V. Berejnov, D. Susac, J. Stumper, A.P. Hitchcock, 3D Chemical Mapping of PEM Fuel Cell Cathodes by Scanning Transmission Soft X-ray SpectroTomography, ECS Trans. 50 (2013) 361-368.

[41] B.V. Derjaguin, V.M. Muller, Y.P. Toporov, Effect of contact deformations on the adhesion of particles, J. Colloid Interface Sci. 53 (1975) 314-326.

[42] G. Strobl, A thermodynamic multiphase scheme treating polymer crystallization and melting, Eur. Phys. J. E. 18 (2005) 295-309.

[43] From the melt via mesomorphic and granular crystalline layers.pdf, https://www.softmatter.uni-freiburg.de/mitarbeiter/strobl.

[44] J. Lyklema, H.P. van Leeuwen, M. van, Cazabat, A. M Vliet, Fundamentals of interface and colloid science, Academic Press, London; Sydney, 1991. 
[45] S. Helmly, R. Hiesgen, T. Morawietz, X.-Z. Yuan, H. Wang, K. Andreas Friedrich, Microscopic Investigation of Platinum Deposition in PEMFC CrossSections Using AFM and SEM, J. Electrochem. Soc. 160 (2013) F687-F697.

[46] R. Hiesgen, I. Wehl, S. Helmly, A. Haug, M. Schulze, A. Bauder, et al., Atomic force microscopy and infrared analysis of aging processes of polymer electrolyte membrane fuel cell components, J. Electroanal. Chem. 662 (2011) 240-250.

[47] M. Watanabe, H. Uchida, Y. Seki, M. Emori, P. Stonehart, Self-Humidifying Polymer Electrolyte Membranes for Fuel Cells, J. Electrochem. Soc. 143 (1996) 3847-3852.

[48] D.K. Paul, K. Karan, A. Docoslis, J.B. Giorgi, J. Pearce, Characteristics of SelfAssembled Ultrathin Nafion Films, Macromolecules. 46 (2013) 3461-3475.

[49] D.K. Paul, K. Karan, Conductivity and Wettability Changes of Ultra-Thin Nafion Films Subjected to Thermal Annealing and Liquid Water Exposure., J. Phys. Chem. C. (2014).

[50] D. Damasceno Borges, A.A. Franco, K. Malek, G. Gebel, S. Mossa, Inhomogeneous Transport in Model Hydrated Polymer Electrolyte Supported Ultrathin Films, ACS Nano. 7 (2013) 6767-6773.

[51] D.D. Borges, A.A. Franco, K. Malek, G. Gebel, S. Mossa, Inhomogeneous transport in model hydrated polymer electrolyte supported ultra-thin films, ACS Nano. 7 (2013) 6767-6773. 